\title{
Trasformazioni sociali, affettivizzazione della sfera pubblica e ri- cerca di senso
}

\author{
Social transformations, affectivization of the public sphere and \\ search for meaning \\ Sergio Salvatore*, Arianna Palmierit, Filippo Pergola***
}

\begin{abstract}
Western societies are crossed by a plurality of critical phenomena. The perception is that of being grappled with an uncontrollable anthropological drift, which make us powerless, leading towards a point of no return. A constitutive character of such a drift is the enslaving of the public spheres to the affects (i.e. affectivization). Any public affair and discussion is less and less addressed in terms of functional criteria (i.e. in terms of optimization of utility in reason of objective data) and more and more as the trigger as well as the target of affective acting-out. Such a dynamics challenges all individuals and institutions that think that civilization and progress are a matter of the human efforts to enslave the affective exercise of the present moment to the capacity of the reason to draw futurables. Education - and higher education within it - is at the front line of this challenge, because it is up to it the effort of promoting the symbolic resources that enables people to succeed in the twofold task of valorizing subjectivity and enslaving its affective substance to aims of social and civic progress. The present paper intends to contribute to address such a challenge. To this end, it proposes an interpretation of the affectivization spreading the contemporary social landscape, based on current debate in cognitive sciences. On this grounds, the general idea that such a phenomenon is something different from and more than a mere epidemy of irrationality - as it is more or less implicitly treated by observers and analysts - is deepened. Following that, strategic and methodological implications for higher education are discussed. It is called for an extension of the function of higher education, in the direction of taking charge of the demand of symbolic resources required to address the uncertainty generated by the contemporary socio-institutional turmoil.
\end{abstract}

Keywords: Social transformation, Affectivization of the publich sphere, Seeking for sense.

\footnotetext{
${ }^{*}$ Professore ordinario di Psicologia dinamica presso l'Università del Salento. E-mail: sergio.salvatore@unisalento.it.

** Professore associato di Psicologia dinamica presso l'Università degli Studi di Padova. Email: arianna.palmieri@unipd.it.

**** Docente a contratto in Psicologia dello sviluppo presso l'Università degli Studi di Roma “Tor Vergata". E-mail: fp.apre@gmail.com.
} 


\section{Introduzione}

Le società occidentali sono colpite da una pluralità di fenomeni critici. Xenofobia, radicalismo religioso e valoriale, violenza razziale e di genere, indebolimento delle infrastrutture civiche, impoverimento del capitale sociale, propagazione di fake news, perdita di fiducia nelle istituzioni nazionali e transnazionali, diffusione di ideologie politiche di estrema destra, sovraniste e populiste, svalutazione della democrazia e delle sue istituzioni, acutizzarsi dei conflitti etnici e internazionali, riemergere dello spettro delle armi nucleari, immobilismo di fronte alla crisi climatica: questi sono solo alcuni dei fenomeni socioculturali e politico-istituzionali che caratterizzano la nostra contemporaneità.

Sebbene stando a quanto riportato da alcuni indicatori statistici le condizioni di vita della popolazione mondiale risultino globalmente migliorate rispetto al passato (ad es. United Nations Development Programme, 2016, cap. 1), l'impressione diffusa è di assistere ad una progressiva deriva antropologica, che ci sta conducendo, impotenti, verso un punto di non ritorno. Un aspetto costitutivo di tale deriva è il dominio assunto, nella sfera pubblica, dagli affetti (d'ora in poi: affettivizzazione). Qualsiasi evento e/o discorso pubblico è sempre meno concepito in termini di criteri funzionali (ad esempio, in termini di ottimizzazione dell'utilità rispetto a dati oggettivi) e sempre più come alimento e/o bersaglio dell'attivazione affettiva. Le persone sembrano essere sempre meno interessate a ricercare soluzioni efficaci che consentano loro di perseguire i loro interessi e proteggere le loro condizioni di vita e sempre più ad utilizzare le circostanze come miccia che inneschi l'esplosione emotiva, da esperirsi nell'immediatezza del momento presente.

Un simile scenario sfida quelle persone istituzioni che concepiscono la civiltà e il progresso come frutto dello sforzo umano di porre l'esercizio degli affetti al servizio della capacità della razionalità di disegnare futuribili. L'istruzione, e l'istruzione superiore più specificamente, è in prima linea in questa sfida, poiché una delle sue principali missioni è di promuovere le risorse simboliche che permettono agli individui di riuscire nel duplice compito di valorizzare la soggettività e vincolarla agli obiettivi del progresso sociale e civile.

Questo articolo si propone di contribuire alla riflessione circa i modi per affrontare tale sfida. A tal fine, di seguito si avanzerà un'interpretazione dell'affettivizzazione dilagante nel panorama sociale contemporaneo, anche sulla base delle attuali acquisizioni nell'ambito delle scienze cognitive. La tesi generale del presente contributo afferma che l'affettivizzazione non possa essere considerata alla stregua di una epidemia di irrazionalità -come, più o meno implicitamente, è stata trattata da critici e analisti; piuttosto, tale fenomeno dilagante è il sintomo di qualcosa di profondamente radicato nel modo di funzionare della mente umana in condizioni di incertezza. Successivamente, verranno discusse 
le implicazioni strategiche e metodologiche che tale linea interpretativa propone per l'istruzione universitaria. In particolare, si discuterà la necessità di un ampliamento della funzione dell'istruzione universitaria, nella direzione della presa in carico dello sviluppo delle risorse simboliche necessarie per affrontare l'incertezza generata dalle turbolenze socio-istituzionali.

\section{Dinamiche socioculturali delle società contemporanee}

\section{L'affettivizzazione della sfera pubblica}

L'indebolimento delle forme razionali del pensiero e lo speculare incremento dell'affettivizzazione della sfera pubblica sono fenomeni evidenti, che assumono varie configurazioni.

In primo luogo, l'affettivizzazione si esprime nei termini della pubblicizzazione del privato, vale a dire della tendenza a rendere i vissuti affettivi attinenti alla sfera delle relazioni primarie oggetto della comunicazione pubblica. Ovviamente, affetti ed emozioni sono sempre stati oggetto del discorso umano; ciò che, tuttavia, oggi è peculiare è la natura autoreferenziale di tale comunicazione. Fino ad un recente passato, l'espressione pubblica del vissuto affettivo è stata generalmente parte di un discorso più ampio - ad esempio, come modo per qualificare il valore di un'azione o di uno scopo (ad es. la rabbia degli oppressi), oppure come materiale su cui letteratura ed arte esercitavano la propria funzione di elaborazione creativa dell'esperienza; oggi, in molte circostanze, la comunicazione degli affetti sembra invece assunta a valore in sé - ovverossia come fine piuttosto che come mezzo. Social media, reality show, trasmissioni televisive dei generi più vari (dai dibattiti politici ai programmi di intrattenimento): sono tutti format accomunati dal loro essere disegnati come occasioni per la mera fruizione del vissuto affettivo.

In secondo luogo, è importante considerare ciò che uno di noi, insieme con altri, ha proposto di definire la nemicalizzazione dell'altro (Salvatore et al., 2018; Mannarini \& Salvatore, 2019), ovvero la rappresentazione affettiva del non familiare/altro da sé come un nemico - un soggetto portatore di intenzionalità aggressiva/distruttiva verso l'agente della rappresentazione e/o il suo gruppo. Pertanto, la nemicalizzazione dell'altro consiste nella generalizzazione e assolutizzazione della categoria amico-nemico come chiave interpretativa dell'esperienza. Tale assolutizzazione produce una peculiare forma di identità, di tipo paranoide: ciò che sostiene e alimenta il legame di appartenenza non è più il senso di partecipazione ad un valore comune (quale può essere, ad esempio, un luogo geografico o simbolico condiviso) ma il sentimento di essere sotto una comune minaccia da parte di un nemico esterno. L'analisi dello scenario 
culturale delle società europee (Salvatore, Fini, et al., 2019; vedi sotto) ha fornito una stima empirica dell'incidenza di questa deriva paranoidea del senso di appartenenza: essa caratterizza il modo di pensare e di sentire di circa $1 / 3$ del campione europeo analizzato (comprendente a 7 paesi: Cipro, Danimarca, Estonia, Grecia, Italia, Olanda, Regno Unito), con una rilevante differenza tra i paesi campionati- dal $22.5 \%$ del campione estone e il $27.1 \%$ di quello danese, al $39.1 \%$ di quello greco e al $40 \%$ di quello italiano.

In terzo luogo, vi è l'irradiazione del legame identitario. Con questo termine intendiamo denotare la tendenza a definire il noi alla luce di oggetti simbolici dematerializzati e mitici - ovvero impoveriti del proprio ancoraggio/radicamento a luoghi, pratiche, interessi e posizioni sociali - e quindi estensibili illimitatamente e asintoticamente, indipendentemente dalle differenze sociali, culturali ed economiche. Il populismo è la forma paradigmatica di questo processo omogeneizzante di irradiazione. Basandoci su una tale definizione, il noi evocato dal populismo è un'entità mitica dotata di una volontà compatta e unitaria, con confini indefiniti e indefinibili. Nella retorica del populismo, il popolo non è un aggregato di individui raggruppati sulla base di un criterio di inclusione (ad esempio, la condivisione della nazionalità rispetto a un determinato Stato); al contrario, chi e cosa costituisce un popolo è tautologicamente definito: l'insieme di coloro che partecipano ad un terreno comune, definito dell'essere contro coloro che ne sono al di fuori.

In quarto luogo, un ulteriore forma dell'affettivizzazione dilagante è data dalla detemporalizzazione della vita sociale. La temporalità - l'idea che ciò che accade si dispiega e trova senso in ragione dell'orizzonte determinato dalla successione più o meno estesa di eventi in relazione non necessariamente lineare tra loro - sembra dissolta come categoria normativa e di senso. Al suo posto, ha acquisito momento la tendenza, caratteristica del modo di operare degli affetti, di assolutizzare il senso e il valore delle azioni e degli eventi sperimentato nell'intorno temporale del loro darsi all'esperienza. La letteratura sui giovani già più di un quarto di secolo fa aveva evidenziato nel presentismo la cifra antropologica delle nuove generazioni (Cavalli \& de Lillo, 1993). A distanza di qualche decennio, dobbiamo registrare come il presentismo sia dilagato, diventando cifra qualificante delle società occidentali nel loro complesso. Un esempio sintomatico di ciò è offerto da quanto è avvenuto nel campo delle lotterie, dove i tempi di attesa tra il momento della puntata e il momento dell'esito sono passati dalla scala delle settimane a quella dei secondi. La perdita di salienza della temporalità è del resto evidente in campo politico ed economico, dove appaiono prevalere logiche di decisione e di azione di corto respiro, cieche rispetto agli esiti paradossali delle scelte compiute (per fare un esempio, il dibattito politico che ha accompagnato l'introduzione in Italia del reddito di cittadi- 
nanza ha quasi del tutto tralasciato di considerare, non fosse altro che per scongiurarlo, il rischio di un impatto regressivo di medio-periodo sulla cultura dei gruppi sociali beneficiari) così come alla loro sostenibilità (in primis ambientale, ma anche sociale, economica) di medio-lungo periodo.

In quinto luogo, la centralità dell'interpretazione affettiva trova riflesso nella evoluzione degli stili e dei canoni del linguaggio pubblico. Dal punto di vista dello stile, è fin troppo ovvio riferirsi alla diffusione, entro la comunicazione sociale e istituzionale, di espressioni insultanti, di volgarità, di denigrazione e di violenza linguistica; meno eclatante, ma forse ancora più indicativa, è la proliferazione di forme retoriche che trovano nell'ancoraggio alla corporeità, al gesto elementare, al concreto, alla visceralità dei simboli primari (in primis religiosi) la propria capacità significante (un esempio, in tal senso, è offerto dal dibattito politico, dove le comunicazioni sono diventate personalizzate, come se si riferissero a relazioni interpersonali, piuttosto che a dinamiche istituzionali). Dal punto di vista del contenuto, è sufficiente richiamare la discussione sulla "post-verità": ciò che viene detto assume valore sempre meno in ragione del proprio contenuto di verità - ad esempio, rispetto al gradiente di conoscenza che lo fonda, della sua utilità, della sua corrispondenza al dato, della sua non contraddittorietà nello spazio e nel tempo - e sempre più come funzione della capacità dell'atto di attivare la risposta affettiva, di operare da oggetto di consumo emozionale e identitario (Codagnone, Bogliacino, Veltri, 2018). Più in generale, la comunicazione pubblica sembra aver messo sullo sfondo il proprio ancoraggio a criteri di funzionalità ed utilità. Il referendum sulla Brexit è un esempio rappresentativo di come il discorso pubblico e le scelte ad esso associate siano al servizio dell'identità. Infatti, nessun elettore aveva le informazioni necessarie per analizzare razionalmente vantaggi e svantaggi funzionali delle due opzioni proposte - rimanere in Europa o meno - dato che le conseguenze per il Regno Unito dell'uscita dall'Unione Europea non erano per nulla prevedibili al momento del voto (Salvatore, Mannarini et al., 2018) ${ }^{1}$.

\footnotetext{
1. Un altro chiaro esempio di come la rappresentazione della realtà possa essere distante da ciò cui si riferisce, proviene dall'Italia, e, più nello specifico, dalla centralità del discorso antimigrazione. Matteo Salvini, nel periodo in cui è stato Ministro degli Interni (Marzo 2018-Agosto 2019), ha descritto i flussi migratori come la principale minaccia da cui l'Italia avrebbe dovuto proteggersi, come un'emergenza in grado di mettere a rischio la sopravvivenza stessa del Paese, tale da giustificare l'adozione di misure radicali, come la chiusura - in violazione del diritto internazionale - dei porti italiani alle navi con a bordo persone salvate dal naufragio. La politica anti-migratoria di Salvini ha avuto un successo straordinario, come dimostra il fatto stesso che la Lega, il partito politico di cui egli è leader, sia risultata essere il primo partito italiano alle elezioni europee del 2019, con il $34.33 \%$ dei voti. Tale risultato è la migliore prova della capacità del discorso anti-migrazione di assecondare e mobilitare gli affetti e dunque dare forma al modo di
} 
In sesto luogo, vi è la dereferenzializzazione del significante (Salvatore \& Scotto di Carlo, 2002), ovvero la perdita della capacità dei segni (parole, immagini) di fornire a colui che li utilizza una "presa" sulle cose cui i segni si riferiscono (Salvatore, 2012). In altre parole, i discorsi non svolgono più la funzione di legare il soggetto al mondo; piuttosto, sempre più spesso essi determinano il loro significato nel loro essere il modo del soggetto di partecipare al legame di appartenenza. I segni si sono impoveriti del loro valore di verità, progressivamente sostituito dal valore socio-affettivo - vale a dire il fatto di essere eventi contingenti agiti in quanto oggetti di immediato consumo emozionale. Più specificatamente, il valore socio-affettivo del segno risiede nella capacità del significante di partecipare alla riproduzione del rituale comunicativo costitutivi del legame di appartenenza. Come accade a Estragone e Vladimiro in attesa di Godot, la parola scambiata è "liberata" dal compito di stare per ciò che è assente, offrendosi così come il luogo ove gli interlocutori sono mantenuti nella loro reciproca presenza.

\section{L'interpretazione prevalente dell'affettivizzazione ed il suo limite}

Molti analisti hanno interpretato le manifestazioni dell'affettivizzazione come reazioni alla Grande Recessione, provocata dal crollo del mercato creditizio americano del 2007, e al conseguente profondo deterioramento delle condizioni di vita di innumerevoli persone (disoccupazione, riduzione dei servizi

pensare e sentire di un ampio segmento della società italiana. Una capacità, questa, priva di qualsiasi sostegno nei fatti, che delineano una realtà molto lontana da quella proposta dalla propaganda:

- nel Giugno 2018 sono arrivati in Italia 3000 migranti; ciò è equivalso ad un calo dell' $87 \%$ rispetto allo stesso mese dell'anno precedente;

- gli stranieri in Italia provenienti da aree extraeuropee sono circa 4 milioni, vale a dire il $6.6 \%$ della popolazione italiana, una percentuale notevolmente inferiore a quella di altri paesi europei - Germania: 8.0\%; Grecia 8.1\%; Francia e Spagna: 8.5\%; Belgio: 8.7\%; Olanda: $8.8 \%$; Austria: $9.9 \%$; Svezia: $11.6 \%$;

- secondo un recente sondaggio (Zanola, 2016) solo un italiano su cinque stima correttamente la percentuale di stranieri nella popolazione italiana. Il $44 \%$ degli italiani pensa che in Italia ci sia il doppio degli stranieri (15\%). Il 33\% degli italiani ritiene che gli stranieri in Italia siano $1 / 3$ della popolazione totale.

In sintesi, la visione della migrazione come un'invasione che minaccia la nostra sopravvivenza e la centralità stessa di una tale rappresentazione nella politica pubblica, non possono essere viste come riflessi di una modalità di pensiero guidata da una ponderazione razionale e obiettiva dei fatti; piuttosto, tale centralità va interpretata come il segno della salienza di altri fattori psico-sociali (e.g. la percezione di incertezza e insicurezza; la necessità di proteggere la propria identità) che asseverano a sé la percezione della realtà (Salvatore \& Freda, 2011): la semantica è stata accantonata ed il suo posto è stato preso dalla dimensione patica del testo. 
pubblici, indebitamento). Altre interpretazioni (ad esempio, Piketty, 2013/2014) hanno posto l'accento sul profondo divario prodotto dalla crescente e drammatica inuguaglianza che colpisce le società occidentali, condividendo, tuttavia, la lettura dell'affettivizzazione come reazione ai cambiamenti delle condizioni economiche. Questa lettura racconta tuttavia solo una parte della storia. La sua parzialità trova evidenza in tre principali elementi.

Primo, l'affettivizzazione del discorso pubblico, se concepita come una reazione alle condizioni materiali, diviene sinonimo di irrazionalità: le reazioni affettive finiscono così per essere affrontate, più o meno implicitamente, come una questione di percezioni errate e di credenze distorte che impediscono alle persone di rappresentarsi lo stato reale dei fatti. Questa prospettiva porta ad assumere un atteggiamento paternalistico nei confronti del punto di vista degli individui - vale a dire, essa conduce all'idea che i sentimenti delle persone, le loro affermazioni e i loro atti siano sbagliati (sebbene venga riconosciuto il fatto che possano essere stati innescati da fattori oggettivi) e perciò devono essere corretti (e/o assecondati); ciò che non si riconosce è che tali sentimenti e atti possano essere compresi in quanto espressione di un punto di vista che, come qualunque punto di vista, possiede la propria valenza di verità. Ancora una volta il dibattito sull'immigrazione in Italia offre un buon esempio di un simile atteggiamento paternalistico (che caratterizza in primo luogo, va riconosciuto, gli osservatori liberali e di sinistra): molti sostenitori di posizioni pro-migrazione implicitamente considerano qualsiasi argomento anti-immigrazione come un sintomo di razzismo e le persone che concordano con le politiche anti-migratorie come ingenue e/o imbarbarite dalla propaganda.

Secondo, deve essere sottolineato che la compresenza di condizioni materiali negative e reazioni affettive diffuse non implica un legame causale di per sé. Questo aspetto è più chiaro se si tiene conto del fatto che in altri momenti storici, condizioni materiali anche peggiori di quelle odierne non si sono associate all'assolutizzazione delle reazioni affettive che qualifica la contemporaneità. Giusto per richiamare un esempio emblematico, in uno dei peggiori momenti della storia contemporanea italiana - l'occupazione nazi-fascista tra il 1943 ed il 1945 - la società italiana fu capace di generare la Resistenza: una risposta collettiva nutrita di passione, coraggio, valorizzazione dell'identità nazionale, ma anche di intelligenza politica e militare, disciplina, senso di cittadinanza e solidarietà (e.g. Battaglia, 1953), ovverossia atteggiamenti e competenze che vedono affetti e razionalità uniti e tra loro sinergici.

Terzo, la visione dell'affettivizzazione come reazione al peggioramento delle condizioni materiali delle persone sottende una visione semplificata della mente e dei processi cognitivi. Infatti, come hanno sottolineato diversi studiosi (e.g. Bruner, 1990; Cole, 1996), le persone non reagiscono alla situazione in sé; piuttosto, tali reazioni riflettono l'interpretazione delle loro condizioni, che si 
esprime nei termini di sentimenti e credenze di incertezza, perdita, dissoluzione degli assetti di vita (Salvatore, Mannarini et al, 2018). Di conseguenza, deve essere riconosciuto che le dinamiche culturali e psico-sociali - ad esempio le dinamiche relative a come le persone danno senso alle proprie esperienze e costruiscono, così facendo, i propri mondi soggettivi (Salvatore, 2018) - possono giocare un ruolo centrale nel plasmare le azioni pensate. Ciò significa che l'interpretazione della realtà sociale è sempre e comunque innescata da condizioni materiali; ma che, una volta innescatasi, essa ha uno spazio di autonomia più $o$ meno ampio rispetto ai dati di realtà. Tale fenomeno avviene sia come conseguenza del fatto che il pensiero individuale e collettivo è vincolato da limitazioni intrinseche nella capacità di prendere in considerazione informazioni, sia del fatto che esso è canalizzato da norme sociali e dagli affetti (vedi sotto, § 3.5; World Bank, 2015; Salvatore, Valsiner \& Veltri, 2019).

In sintesi, l'impatto degli affetti sul pensiero non può essere visto come il fallimento di un modello ideale di cognizione che la identifica con la sua componente di computazione razionale: al contrario, va riconosciuto che gli affetti sono parte integrante della cognizione e contribuiscono in modo essenziale allo sforzo del sistema cognitivo di mappare la realtà nel modo più funzionale possibile. Servono dunque modelli che permettano di comprendere la sinergia tra le componenti razionali ed affettive dei processi cognitivi. Tali modelli sono necessari per poter andare oltre l'approccio descrittivo che si limita a rilevare la mera compresenza di fattori materiali e reazioni affettive, così da comprendere perché le persone rispondono all'incertezza dirompente tramite l'affettivizzazione della sfera pubblica. Solo una profonda comprensione del meccanismo causale sotteso all'affettivizzazione della sfera pubblica può consentire alle istituzioni e agli attori della politica e dell'educazione di progettare strategie efficaci di contrasto delle manifestazioni critiche di tale fenomeno.

\section{Affetti ed incertezza}

Un primo passo nella direzione di un modello dell'affettivizzazione dell'attuale contesto socio-politico può essere compiuto facendo riferimento all'interpretazione generale dello stesso come modo di fronteggiare cognitivamente la condizione di radicale incertezza e di perdita di senso che caratterizza la società contemporanea (e.g. Mazzoni, 2015).

Secondo questa chiave di lettura, il ricorso all'interpretazione affettiva dell'esperienza è il modo con cui il soggetto mantiene significativa la relazione con un mondo la cui elevata instabilità e mutevolezza rende obsoleti i codici di senso (e.g. saperi, valori, significati istituiti, norme, copioni comportamentali) posti a fondamento delle forme di vita consolidate. Allo stesso tempo, il ricorso 
agli affetti, se da un lato offre la possibilità di recuperare un ancoraggio stabile all'attività interpretativa (cfr. § 3-5), dall'altro indebolisce i meccanismi della regolazione sociale e dunque in ultima istanza incentiva ulteriormente le dinamiche di fondo che determinano le condizioni della perdita di senso.

Questa prospettiva interpretativa ha radici profonde nelle scienze sociali. Il nesso tra modernizzazione/sviluppo socio-economico e perdita di senso è al centro della riflessione di autori classici, quali Durkheim (1893/1984) e Thomas e Znaniecki (1919-1920). La letteratura psicosociale degli ultimi venti anni ha ulteriormente approfondito il tema, focalizzandosi sugli effetti socio-cognitivi della incertezza ambientale. Nel suo complesso, tale letteratura è unanime nell'evidenziare l'impatto destabilizzante che (in senso lato) l'instabilità ambientale esercita sul funzionamento cognitivo. Le teorie divergono tuttavia in come definiscono e spiegano il fenomeno: diversi sono i modi con cui sono concettualizzati la causa (i.e. che cosa della condizione ambientale agisce come fattore destabilizzante), l'effetto psicologico (i.e. su che cosa la destabilizzazione agisce), così come i sottostanti meccanismi causali (i.e. in ragione di quale processo un determinato fattore destabilizzante produce l'effetto che produce). In estrema sintesi, alcune teorie propongono spiegazioni di tipo funzionale - vale a dire, spiegazioni basate sull'idea che l'incertezza indebolisce la funzionalità del sistema cognitivo, in termini di capacità di inferenza causale (Control Uncertainty Model, Weary; Tobin \& Edwards, 2010) e/o della regolazione del comportamento finalizzato (Defensive Approach Motivation; Marigold, Mc Gregor, \& Zanda, 2010) o del controllo della relazione con l'ambiente (Compensatory Control Theory; Kay et al., 2009) e che le risposte dell'individuo sono finalizzate a recuperare tale deficit di funzionalità; altre teorie leggono l'incertezza in termini di minaccia alla stabilità del sé e dell'autostima (Terror Management Theory, Greenberg \& Arndt, 2012; Self-Uncertainty Management Model; Sedikides et al., 2010; in parte Uncertainty Management Model; van Den Bos \& Lind, 2010), focalizzandosi di conseguenza su effetti che riflettono il modo con cui le persone cercano di proteggere il proprio senso di sé; un altro gruppo di teorie interpreta invece la destabilizzazione in termini di significato - vale a dire come violazione del sistema di credenze/visione del mondo con il quale il soggetto si identifica (Meaning Manteinance Model, Proulx, \& Inzlicht, 2012; Worldview Verification theory; Major et al., 2007).

Una comprensione più sistematica e approfondita dei meccanismi che rendono psicologicamente destabilizzante l'esposizione all'incertezza ambientale, così come del ruolo che i fattori individuali, micro e macro-sociali giocano in tale dinamica, richiede un'integrazione tra tali tre approcci, così da portare a sintesi gli aspetti della complessità del fenomeno che ciascuno di essi evidenzia. È questo un obiettivo scientifico di notevole rilievo, non solo per ragioni teoriche ma anche perché, come sopra detto, su di esso si basa la definizione di 
strategie di intervento volte a contrastare la pervasività dell'affettivizzazione entro le dinamiche socio-culturali della contemporaneità. Nei prossimi due paragrafi verrà delineato un modello generale delle dinamiche che sottostanno alla relazione tra incertezza e affettivizzazione, al cui sviluppo e validazione empirica gli autori di questo articolo, con altri, stanno lavorando.

\section{Concetti di base}

In questa sezione presenteremo i concetti alla base del modello dell'incertezza-affettivizzazione, delineato nel successivo paragrafo. Tali concetti provengo da due principali fonti teoriche: la Psicologica Culturale-Semiotica (PCS, e.g. Salvatore, 2018; Valsiner, 2014) e la Embodied Cognition (e.g. Barsalou, 1999; Lindblom, 2015)

\section{Ifondamenti: mediazione e triadicità del segno}

I pilastri della discussione che segue la mediazione e la triadicità del segno - sono due principi che la PCS condivide con varie scuole di pensiero attive entro le scienze sociali (ad esempio Bruner, 1990; Berger \& Luckmann, 1966; Vygotsky, 1974).

Il principio della mediazione pone i significati e il processo di costruzione degli stessi al centro della modellizzazione dei processi cognitivi (Salvatore, 2018). Tale principio afferma che i processi cognitivi sottesi al pensiero e all'azione sono funzione dell'interpretazione della realtà (piuttosto che essere funzione della realtà in sé), e che tale interpretazione è modellata e vincolata al sistema di significati del soggetto. Pertanto, il significato media tra il soggetto e il mondo, come una lente che modella e limita ciò che un individuo vede - le lenti non creano ciò che una persona percepisce; piuttosto, ne definiscono la forma. Questo significa che le persone non sono irrazionali; piuttosto, il modo in cui rappresentano la realtà dipende dal significato attribuito ad essa; il significato fa da cornice per l'interpretazione della situazione.

Il principio della triadicità del segno si riferisce al modello semiotico di Peirce (e.g. Peirce, 1897/1932). Esso completa il precedente principio fornendo una definizione di ciò in cui il significato consiste. Secondo la teoria della triadicità del segno, il significato è la capacità del segno di elicitare una risposta nell'interprete, vale a dire un ulteriore segno ("creare nella mente della persona un segno equivalente, o, forse, più sviluppato"; Peirce, 1897/1932, Vol. 2, p. 
238 " nostra traduzione dall'originale inglese), che stabilisce "sotto quale profilo o capacità" il segno "sta per" il segno precedente (Peirce, 1897/1932, Vol. 2 , p. 238). La triadicità consiste in questo: il segno non si esaurisce nella relazione tra un significante e l'oggetto cui si riferisce: esso implica un terzo elemento: la funzione interpretativa dell'interprete.

Ciò che è rilevante notare è il fatto che la relazione triadica procede senza interruzioni: il segno innesca un nuovo segno nella mente dell'interprete che, a sua volta, innesca un ulteriore segno interpretante, e così via. Il significato emerge dunque da una catena semiotica infinita, entro la quale ogni elemento è al contempo l'interpretazione della sequenza precedente, la sua realizzazione nel momento presente e l'elicitatore del segno successivo, che proietta la sequenza in avanti nel tempo. In altri termini, il segno è dotato di una doppia valenza, retroattiva e proattiva: da un lato, ciò che segue è la risposta interpretativa che il segno precedente elicita nella mente dell'interprete; dall'altro, è l'innesco che attiva/orienta la selezione del segno successivo (Salvatore, $2016)^{2}$. In sintesi, il principio di triadicità del segno concepisce il significato come la funzione di relazione del segno, vale a dire la distribuzione di probabilità che definisce la sua tendenza a co-occorrere (i.e. a seguire e a precedere) con gli altri segni (Proulx \& Inzlicht, 2012; Salvatore, 2018).

\section{Gli affetti sono segni}

In questo paragrafo sarà approfondito un corollario della triadicità dei segni: la visione degli affetti come segni.

\footnotetext{
${ }^{2}$ Ad esempio, si immagini una persona che guardando il cielo azzurro sgombro di nuvole, pensi: "Oggi non pioverà" e successivamente: "posso andare al mare come avevo programmato". Il segno "oggi non pioverà" è un'interpretazione del segno precedente (il cielo sgombro di nuvole); tale segno viene interpretato nei termini della qualità per cui esso sta, vale a dire la qualità di non volgere a pioggia. A sua volta, tale pensiero elicita un successivo pensiero che opera da segno che interpreta retroattivamente il precedente, nei termini della attribuzione alla qualità di non piovere della qualità di permettere la conservazione del programma desiderato. A completamento dell'esempio, sono utili due osservazioni. Da un lato, va tenuto conto che la catena semiotica può tanto essere prodotta da un unico individuo, come sequenza di pensieri o parole del singolo, tanto dalla cooperazione tra più persone; ciò che conta è che un segno successivo prolunghi la sequenza, indipendentemente da quale ne sia la fonte. In secondo luogo, va evidenziato che le successive interpretazioni possono tanto operare da riproduzione della qualità magnificata dalla precedente interpretazione, che da specificazione-modulazione, riscrittura più o meno radicale di essa. L'esempio proposto riguarda il secondo caso. Un esempio del primo caso è dato da quelle situazioni in cui una rappresentazione successiva opera da mappa del significato della precedente - ad es. "questo oggetto è una penna" - "cioè, uno strumento per scrivere". Un esempio del terzo caso sono le battute umoristiche che nella fase preparatoria fan sì che si consolidi una linea interpretativa per poi violarla con la frase finale.
} 
Come lo stesso Peirce afferma, ciò che qualifica il segno non è la materia in cui esso consiste (parola, immagine, ecc.) ma la sua capacità di entrare nella catena semiotica, con la doppia valenza di interpretante del segno precedente e elicitatore del segno successivo che andrà a sua volta ad interpretarlo ${ }^{3}$.

Una implicazione centrale di tale visione è che gli affetti vanno considerati segni alla stessa stregua delle parole e delle immagini (Salvatore \& Zittoun, 2011). Per quanto fenomenicamente differenti, una parola, una foto, una formula matematica, un gesto, una reazione affettiva, possono tanto essere quanto non essere segni, ciò dipendendo non dalla loro natura intrinseca ma dalla funzione che assolvono o meno nella contingenza del processo di significazione ad es. una parola in una lingua sconosciuta all'interprete, dunque non riconosciuta come forma lessicale, non è un significante di cui non si comprende il significato; piuttosto, è un elemento inerte dal punto di vista semiotico (almeno fin quando non è riconosciuto come "parola di una lingua sconosciuta" - in tal caso tale riconoscimento opererebbe da segno interpretante). Provare un moto di stizza all'autogoal subito dalla squadra del cuore non è una mera reazione emozionale conclusa in sé stessa ma un atto di significazione operato tramite la mobilitazione di un segno corporeo: una risposta affettiva che interpreta la catena semiotica precedente nei termini dello stato di attivazione neurofisiologica, al contempo predisponendo il corpo alla produzione di altri segni (ad es. l'insulto nei confronti del difensore autore dell'autorete).

Quanto sopra detto ci permette di giungere ad una conclusione. La concezione triadica del segno come funzione di relazione si offre come una definizione che unifica i diversi tipi di rappresentazione (simboli astratti, icone, risposte motorie) impiegati dalla cognizione umana, ad un livello sovraordinato rispetto alle loro pur rilevanti differenze fenomeniche e funzionali.

Questa conclusione è rilevante da un punto di vista concettuale. Essa permette di riconoscere la linea di continuità che accomuna affetti e pensiero astratto, in quanto tipi diversi di segni. Ciò rende possibile la definizione di un modello volto a spiegare come essi operano, fornendo al contempo il modo di connettere i livelli individuale e sociale di analisi del processo di costruzione del significato (vedi sotto, §3.5).

\footnotetext{
${ }^{3}$ In proposito, è utile ritornare all'esempio proposto in nota 2 . Il cielo azzurro sgombro di nuvole opera da segno in quanto il segno successivo lo rende parte di una catena semiotica; in molte altre circostanze lo stesso cielo non opera da segno per la semplice ragione che non viene semiotizzato - vale a dire, non viene seguito da un segno che lo interpreta. Inoltre, va evidenziato che la possibilità di essere o meno semiotizzato non dipende dalle caratteristiche dell'evento/oggetto, ma dall'interprete, dalla sua relazione contingente con il mondo: dal suo progetto semiotico (e.g. dal fatto che il soggetto dell'esempio ha a cuore un programma rispetto al quale le condizioni atmosfere avverse assumono rilievo).
} 


\section{La natura embodied del significato}

La visione degli affetti come segni proposta dalla Psicologia Semiotico-Culturale (di seguito: PSC) è coerente con la teorizzazione della natura embodied della cognizione sviluppata dalla psicologia nel corso degli ultimi 20-25 anni (ad esempio Barsalou, 1999, 2016; Borghi et al., 2017; Cuccio \& Gallese, 2018). Secondo l'Embodied Cognition, i significati nei termini dei quali le persone interpretano l'esperienza sono di natura corporea, piuttosto che simboli immagazzinati nella mente. In altre parole, le rappresentazioni del mondo - il significato attribuito all'esperienza di esso - non sono di natura astratta - amodale, secondo la terminologia adottata (Barsalou, 1999); al contrario, le rappresentazioni sono radicate negli stessi circuiti neurali sottesi all'elaborazione sensoriale degli stimoli - in altri termini, sono fatti della stessa sostanza neurobiologica delle sensazioni, costituiti da pattern senso-motori entro i quali percezione e risposta motoria sono intrecciate e difficilmente separabili sul piano funzionale. Ciò significa che la conoscenza concettuale del mondo in ultima istanza consiste nelle configurazioni senso-motorie, modellate attraverso le interazioni ricorsive con l'ambiente, che definiscono la propensione del corpo (con il cervello parte di esso, ovviamente) ad agire in un certo modo in rapporto ad oggetti e situazioni. In questo senso, si potrebbe dire che il soggetto non ha ma è conoscenza (Verheggen, \& Baerveldt, 2007) - la rappresentazione è la forma dinamica dell'essere nel mondo.

Da quanto sopra detto, discende che la visione embodied della mente implica l'idea che i processi cognitivi - a partire dalla, ma non limitatamente alla, percezione - siano situati, vale a dire sono al servizio dell'azione (Engel, Friston, $\&$ Kragic, 2015). Ciò significa che il soggetto non è un meccanismo computazionale che indipendentemente dal corso dell'azione elabora la rappresentazione della situazione che successivamente utilizza per regolare la propria risposta. Al contrario, le rappresentazioni sono pattern dinamici (i.e., processi che si modificano nel tempo) di attività senso-motoria nei termini della quale il corpo mantiene la propria azione accoppiata con il mondo, come nel caso di due ballerini che riproducono la propria danza nel susseguirsi del tempo presente: il ballerino non definisce una rappresentazione del piano dei propri movimenti e dei movimenti del partner per poi eseguirlo; se così fosse, non avrebbe il tempo per utilizzare i feedback relativi ai micro-scostamenti tra piano e risultato, necessari per modulare il comportamento. Al contrario, il ballerino si rappresenta il movimento della propria danza nei termini del proprio stesso movimento, delle micro-variazioni istantanee che realizza per rispondere alle destabilizzazioni locali della reciproca coordinazione. In altri termini, la cognizione opera "on-line": è il modo del soggetto di essere presente nella contingenza delle situazioni. 
La natura inferenziale del processo di costruzione del significato (meaningmaking)

La natura embodied e situata della cognizione ed il connesso riconoscimento del carattere on-line della cognizione hanno un corollario rilevante ai fini della presente discussione: la natura inferenziale del processo di significazione. Il punto da considerare è che la situazione ambientale è intrinsecamente dinamica - ovverossia, è un insieme di occorrenze che tendono a variare istante per istante. Di conseguenza, le micro-variazioni senso-motorie necessarie per mantenere il corpo accoppiato con la situazione devono attivarsi in tempi rapidissimi, altrimenti, data la rapidità della variazione ambientale, esse risulterebbero superate già al momento del loro realizzarsi. Ciò significa che la micro-regolazione senso-motoria non può avvenire sulla base di un meccanismo di retroazione che agisc una volta che la risposta comportamentale è stata prodotta, sulla base del suo esito. Se così fosse, la correzione permessa dal feedback si riferirebbe ad uno stato ambientale già appartenente al passato; il sistema cognitivo si troverebbe in una condizione di perenne, vana rincorsa. È, dunque, opportuno assumere che la micro-regolazione si realizzi nello stesso intorno temporale della variazione della situazione che la induce (per inciso, tale affermazione non è un ossimoro, in quanto il tempo presente ha comunque una durata, sia pure misurabile in centesimi di secondo; cfr. Salvatore, 2016; Stern, 2004; Varela \& Depraz, 2000), sulla base di un meccanismo di inferenza bayesiana (Barsalou, 2011) $)^{4}$. Secondo tale tesi, il corpo non regola il proprio stato in maniera reattiva rispetto allo stato attuale della situazione, ma in modo anticipatorio, in funzione della previsione dello stato ambientale del momento successivo (l'inferenza è concepita come bayesiana per evidenziare che la previsione è condizionale, vale a dire: basata sull'esperienza pregressa delle traiettorie evolutive dell'ambiente).

\footnotetext{
${ }^{4}$ La visione inferenziale della cognizione trova la sua radice filosofica nel concetto di intenzionalità di Husserl (1919/1991; per una discussione, si veda Gallagher, 2005). Essa è coerente con le recenti scoperte neuroscientifiche relative ai neuroni specchio (Cuccio \& Gallese, 2018). $\mathrm{Al}$ di là delle interpretazioni meta-teoriche e metaforizzanti che di tale meccanismo neurale sono state offerte (per inciso, non sempre in modo circostanziato), i neuroni specchio sono una delle prove empiriche più chiare della natura inferenziale della percezione. Questo tipo di neuroni si attiva tanto quanto la scimmia compie un'azione che quando la osserva in un altro. Ciò significa che il pattern motorio si attiva non solo per regolare l'azione in atto ma anche come simulazione motoria di una percezione (i.e. la percezione dell'azione che un altro individuo compie). In definitiva, i neuroni specchio mostrano che la percezione di un'azione almeno parzialmente si realizza nei termini dell'attivazione di una risposta motoria che simula/attiva l'azione associata alla percezione.
} 
Più precisamente, il sistema cognitivo embodied inferisce, a partire da indizi sensoriali, il pattern sensoriale che mappa lo stato ambientale del momento successivo e completa tale pattern sensoriale con la simulazione della risposta motoria che ottimizza il fit con esso - ovverossia, il successo della previsione. $\mathrm{Nel}$ momento successivo (che è il momento in cui effettivamente si realizza lo stato ambientale anticipato dalla inferenza percettiva operata nel momento precedente), da un lato, il sistema cognitivo riscontra l'eventuale perdita di fit e, nel caso, modula la risposta; dall'altro lato, produce un'ulteriore inferenza che proietta il meccanismo previsionale al momento successivo.

In definitiva, la modalità inferenziale aumenta notevolmente l'efficienza e l'efficacia della regolazione, rendendola adeguata alla sua funzione di rendere possibile "l'accoppiamento on-line" dell'azione con l'ambiente, in quanto permette di controllare momento per momento il processo lungo il suo corso, piuttosto che in termini di verifica post hoc dell'esito dell'azione. ${ }^{5}$

Un corollario della natura inferenziale della cognizione è la sua autoreferenzialità. Con questo concetto intendiamo fare riferimento al fatto che i processi cognitivi funzionano secondo la regola fondamentale di investire di significato una determinata esperienza (Valsiner, 2007). Ad un livello fenomenologico, tale attribuzione di significato si basa sul fatto che il soggetto che produce significati (il meaning-maker) è capace di generare interpretazioni della realtà che gli/le permettono di rimanere ancorato/a al mondo (Maturana \& Varela, 1980). Ad un livello di analisi computazionale, l'attribuzione di significato può essere vista come la capacità del sistema di significati del soggetto di riprodursi

\footnotetext{
${ }^{5}$ Si pensi ad un tennista: se per regolare il colpo dovesse attendere l'esito dell'impatto della racchetta sulla pallina, allora verrebbe meno la possibilità stessa di regolare l'azione nel corso del suo prodursi, riducendosi il controllo alla possibilità di riconoscere post hoc la riuscita o meno dell'esecuzione implementata. In realtà, il tennista monitora una serie di indizi ambientali ancor prima che la palla sia stata colpita dall'avversario (e.g. monitora la posizione del corpo di questi, come sta impugnando la racchetta, l'angolo e il rimbalzo della palla) e su tale base produce un'inferenza percettiva dello stato ambientale successivo (e.g. come l'avversario colpirà la palla), consistente nell'anticipazione delle sensazioni che stanno per realizzarsi (e.g. il rumore del colpo, la variazione della posizione dell'avversario, l'angolatura della racchetta dell'avversario, così come velocità e posizione della palla in uscita dal colpo); allo stesso tempo tale mappa inferenziale elicita la risposta motoria (e.g. un lieve spostamento della testa e degli occhi, una variazione della posizione e uno spostamento sul campo, tutto finalizzato alla ricerca del fit della percezione della traiettoria) che ottimizza il mantenimento della coerenza tra lo stato inferito e lo stato sensoriale percepito al momento successivo (i.e. il fit). La nuova inferenza, relativa al momento successivo, si baserà sullo stato del fit - maggiore lo scostamento tra inferito e percepito, maggiore la necessità dell'inferenza di incorporare variazioni del pattern senso-motorio volte a recuperare il fit (ad esempio, se l'avversario produce una finta o un colpo in contropiede, tale cambiamento improvviso si tradurrà nella improvvisa rilevazione di una perdita di fit tra traiettoria attesa e percepita, che indurrà una variazione ampia della risposta motoria, ad esempio una variazione nella direzione della corsa).
} 
mantenendo il fit della propria inferenza (Salvatore, 2019). Di seguito proponiamo di definire tale capacità come il valore del segno (cfr. §4.1). È importante sottolineare che questo principio non nega che i processi cognitivi possano essere basati sulle informazioni raccolte dal mondo esterno - in altri termini, non nega che i processi cognitivi seguano una regola di mappatura razionale della realtà; tuttavia, aggiunge un'ulteriore componente, ovverossia il riconoscimento del fatto che la razionalità lavora in una sorta di interazione dialettica con la necessità del sistema cognitivo di mantenere stabile nel tempo il sistema di significati su cui fonda il proprio funzionamento. Primum vivere è la regola fondamentale (ma non l'unica) cui si attiene il sistema cognitivo.

\section{L'incorporazione del meaning-making nella cultura. Universi simbolici}

La PSC evidenzia che i segni affettivi fondano e modellano significati generalizzati - visioni del mondo - che sono alla base del modo in cui gli esseri umani danno senso al loro essere nel mondo. Tali sistemi sono credenze globali latenti sul sé e sulla realtà, riconoscibili solo indirettamente, attraverso gli output cognitivi e pragmatici che mediano (ad esempio in termini delle affermazioni, valutazioni, azioni su questioni specifiche che producono).

Come detto, gli affetti fondano e modellano tali visioni del mondo. Le fondano, nel senso che le visioni del mondo vengono caricate del valore soggettivo attraverso il loro ancoraggio alle esperienze affettive legate all'attivazione del corpo; le modellano, nel senso che ciascuna visione del mondo è un significato incarnato che consiste in una propensione a relazionarsi in un certo modo con la realtà, modo configurato dal pattern affettivo ad essa associato ${ }^{6}$. Da un punto di vista complementare, gli affetti rendono pertinenti certe specifiche componenti della relazione con il mondo e in tal modo limitano l'elaborazione cognitiva dell'esperienza alla variabilità ambientale che riguarda tali componenti' .

\footnotetext{
${ }^{6}$ Ad esempio, uno stato affettivo di rabbia favorirà la generazione di una visione del mondo costituita da una propensione ad entrare in rapporto con lo stesso nei termini di qualcosa di negativo da attaccare e distruggere.

${ }^{7}$ Ad esempio, l'affetto piacere/dispiacere rende pertinente la componente del mondo che corrisponde alla variabilità della presenza/assenza della sua capacità di conservare lo stato stazionario (i.e. la piacevolezza). Di conseguenza, il meaning-maker, con il proprio affetto, tenderà a mettere in primo piano tale variabilità - vale a dire a ricercare i segni della presenza o assenza delle condizioni di piacevolezza; in altri termini, a rappresentare il mondo sotto il profilo del suo proporsi come buono (in caso di presenza) o cattivo (in caso di assenza).
} 
Sulla base del framework teorico della PSC, Salvatore e colleghi (2018) hanno concettualizzato tali visioni del mondo nei termini di universi simbolici ${ }^{8}$. Gli universi simbolici rappresentano sistemi intituivi di assunzioni che mediano l'attività interpretativa delle persone. La natura affettiva degli universi simbolici è visibile da quattro delle loro principali caratteristiche. In primo luogo, gli universi simbolici rappresentano una credenza riguardo il sé e il mondo che non descrive le cose come sono, ma come ci si aspetta che siano. Questo significa che un universo simbolico è il fondamento del processo di inferenza alla base della costruzione di significati; da un altro punto di vista, esso è il modo in cui il soggetto si posiziona nel mondo ${ }^{9}$. In secondo luogo, gli universi simbolici sono olistici,_vale a dire essi sono credenze globali che comprendono l'intera esperienza della relazione con il mondo, piuttosto che singole parti della stessa (e.g. eventi specifici, oggetti o domini della vita). In terzo luogo, gli universi simbolici non riflettono la realtà - nel senso che non sono vincolati a mappare la complessità del mondo; piuttosto, ciascun universo simbolico seleziona un aspetto peculiare della realtà - un sottoinsieme di relazioni tra gli infiniti elementi del campo dell'esperienza - e lo generalizza/assolutizza, trasformandolo in regola universale, definitoria di come le cose funzionano ${ }^{10}$. In quarto luogo, coerentemente con il punto precedente, gli universi simbolici non rispettano $\mathrm{i}$

\footnotetext{
${ }^{8}$ Una recente analisi del contesto culturale delle società europee (Salvatore et al., 2018) ha identificato 5 diversi universi simbolici:

1. Universo ordinato. Al centro di questa visione del mondo vi è una concezione positiva onnicomprensiva dello stesso (istituzioni, servizi, futuro, persone), percepito come affidabile e fondato sull'identificazione di valori trascendenti (e.g. giustizia, solidarietà ecc.);

2. Legami interpersonali. I legami interpersonali primari sono visti come l'unica dimensione significativa della vita.

3. Società che si prende cura. Tale universo simbolico concepisce la società e le istituzioni come responsive ai bisogni individuali e perciò, in grado di alimentare la capacità delle persone di perseguire progetti e scopi;

4. Nicchia di appartenenza. Tale visione connotano il mondo come un posto inospitale e minaccioso: il gruppo di appartenenza è il rifugio dove trovare protezione;

5. Il mondo degli altri. Tale universo simbolico esprime una visione completamente negativa e disperata del mondo, caratterizzata da un senso generalizzato di sfiducia, disperazione e impotenza; il mondo appartiene agli altri, ai vincitori che hanno il potere.

${ }^{9}$ Ad esempio, connotare affettivamente qualcosa come buono, significa l'esperirlo come un amico, vale a dire come il destinatario di un'azione cooperativa.

${ }^{10} \mathrm{Si}$ consideri ad esempio una persona innamorata. Il campo di esperienza di tale persona è ridotto agli elementi che concernono il senso di piacevolezza fornito dall'esistenza dell'amato; tale componente del reale è assolutizzata -i.e. è l'unico aspetto che conta - e generalizzata - i.e. è la regola interpretativa che media l'esperienza dell'intera relazione con il mondo (ad esempio, cosa accade a lavoro, nelle relazioni con gli amici e così via).
} 
vincoli semantici: ciascun universo simbolico unisce credenze che possono tanto avere quanto no coerenza semantica l'una con l'altra ${ }^{11}$.

La natura affettiva degli universi simbolici ha due implicazioni rilevanti che è importante che vengano sottolineate in questa sede.

Innanzitutto, essa costituisce la ragione per cui sono condivisi all'interno di gruppi sociali e, così facendo, possano operare da significati culturali che legano (canalizzando, vincolando e finalizzando) il processo cognitivo individuale con l'ambiente sociale. Ogni universo simbolico è condiviso da un da un certo sottogruppo di persone non attraverso la negoziazione, ma in virtù del fatto che le persone hanno le stesse modalità di base di funzionamento degli affetti (ad esempio, il corpo di tutti gli esseri umani - allo stesso modo di tutti gli altri esseri viventi collocati al livello superiore del continuum filogenetico è caratterizzato da un pattern affettivo che include lo stato neuro-fisiologico di piacevolezza/spiacevolezza). In altre parole, ciascun universo simbolico consiste in un pattern affettivo che emerge dall'interazione con una certa configurazione di esperienza. Pertanto, dato che la variabilità interindividuale dei modelli affettivi è limitata, le persone che interagiscono con una certa configurazione di esperienza tenderanno ad essere relativamente simili circa l'universo simbolico di riferimento.

La seconda implicazione della valenza affettiva degli universi simbolici è la sua natura performativa. Gli universi simbolici che fondano e canalizzano le dinamiche culturali sono riprodotti nel tempo e all'interno di gruppi sociali attraverso il loro essere messi in atto - vale a dire, attraverso il fatto che vengano usati come presupposti per fondare credenze, azioni e scelte. In altre parole, le dinamiche culturali di costruzione del significato non richiedono - al livello profondo dei significati affettivi - accordo consensuale; piuttosto, gli universi simbolici che modellano le manifestazioni culturali sono riprodotti - e possono svilupparsi - proprio a causa (e in termini) del loro essere incorporati nelle pratiche sociali. In breve, mentre da un lato gli universi simbolici rendono possibili e modellano le pratiche sociali, dall'altro lato, tali pratiche veicolano i gli universi simbolici su cui si basano.

\section{Affetti ed incertezza}

Il modello embodied e semiotico del funzionamento mentale delineato nel

\footnotetext{
${ }^{11}$ Ad esempio, a livello della significazione affettiva, se una cosa è bella, essa tenderà ad essere percepita come anche buona ed affidabile, sebbene non vi sia alcun legame semantico tra queste caratteristiche (Tonti \& Salvatore, 2016).
} 
precedente paragrafo offre una chiave interpretativa per comprendere il rapporto tra incertezza ed attivazione affettiva, che si presta a sua volta ad interpretare l'affettivizzazione che caratterizza la vita sociale e istituzionale contemporanea.

\section{Il valore semiotico}

In via preliminare, precisiamo l'uso che faremo di seguito della nozione di valore semiotico (in alcuni casi, di seguito solo: valore). Con tale termine si vuole qui denotare la capacità di un segno di elicitare una risposta senso-motoria che mantiene l'azione accoppiata con l'attività in corso. Il valore di un segno è dunque analogo al valore di una moneta, inteso come la sua capacità di scambio - ovverossia, in termini più astratti e coerenti con la terminologia usata in questa sede, inteso come la capacità di legame che la moneta instaura con ciò per cui sta (i.e. l'oggetto della transazione economica). Da un punto di vista più specifico, dato il carattere inferenziale della cognizione, il valore del segno consiste nella sua efficacia nel sostenere la capacità di fit dell'inferenza bayesiana operata del sistema cognitivo ${ }^{12}$.

\section{L'organizzazione degli stati corporei e la multidimensionalità del valore}

Quanto detto sopra a proposito del valore semiotico del segno va integrato da un'ulteriore osservazione relativa alla natura multidimensionale del valore del segno. Tale caratteristica dipende dalla peculiare organizzazione degli stati senso-motori. Da questo punto di vista si possono distinguere concettualmente almeno cinque livelli di organizzazione del funzionamento dell'organismo.

Al livello di base (che definiamo livello 0), troviamo i meccanismi neurofisiologici che veicolano il funzionamento dell'organismo (e.g. respirazione, battito cardiaco). Tali meccanismi hanno un proprio modo di esercizio suscettibile di modulazione in ragione delle contingenze ambientali, dunque degli ambiti di attività associate a specifici cluster di tali contingenze (ad es. nel contesto dell'attività del correre il battito cardiaco accelera). La capacità dei meccanismi

\footnotetext{
12 Secondo questa prospettiva, la funzione di relazione in cui il segno consiste può essere interpretata come la probabilità a priori che determina $i$ valori della probabilità condizionale dell'inferenza bayesiana: maggiore è la capacità del segno di rappresentare un determinato ambito di attività, discriminandolo rispetto agli altri, maggiore il suo contributo alla inferenza, come conseguenza della possibilità di restringere lo spettro delle traiettorie evolutive del campo di esperienza a quelli compatibili con l'ambito di attività in gioco.
} 
di base di variare il proprio funzionamento è alla base del carattere stratificato dell' organizzazione dell' organismo vivente (il che è un altro modo per dire che un organismo vivente è un sistema cognitivo, cfr. Maturana e Varela, 1980). Un primo livello di organizzazione (livello l) è dato dallo strutturarsi di pattern generalizzati di co-varianza tra i meccanismi neurofisiologici di base. È questo il livello degli affetti, da intendersi come dimensioni bi-polari, ciascuna strutturata nei termini della giustapposizione di due configurazioni globali dei processi neurofisiologici (Barrett Feldman, 2006). Ad esempio, la dimensione affettiva piacevole/spiacevole consiste nella giustapposizione tra, da un lato, uno stato caratterizzato dalla co-occorrenza di modi di funzionamento dei meccanismi neurofisiologici che nel loro insieme (o quasi) mantengono il corpo in una condizione stazionaria, di non necessità di cambio della condizione data e, dall'altro lato, uno stato opposto consistente nella co-occorrenza di modi di funzionamento dei meccanismi neurofisiologici che qualificano la condizione globale del corpo come necessitante di modifica di stato. Potente/debole, attivo/passivo, interno/esterno sono altre dimensioni affettive rintracciabili in letteratura (e. g. Osgood, May \& Miron, 1975; Osgood, Suci \& Tannenbaum, 1956). Ad un livello di organizzazione di maggiore differenziazione (livello 2) troviamo le forme di attivazione del corpo che riguardano l'esecuzione di condotte guidate dalle affordance ambientali (ad es. sollevare, camminare, masticare, ecc.), vale a dire dalle caratteristiche dell'ambiente che rendono praticabile una certa modalità di relazionarsi dell'organismo (ad esempio, una sedia esprime l'affordance del sedersi; cfr. Gibson, 1979). Ciascuna di queste condotte consiste in un cluster di variazioni dinamiche di una sottoclasse dei processi neurofisiologici di base. Si tratta, dunque, di configurazioni "specializzate" rispetto alle dimensioni affettive. Inoltre, a differenza di quest'ultime, esse sono discrete, nel senso che non si definiscono in giustapposizione da altre configurazioni, ma in quanto cluster di co-occorrenze di stati senso-motori e loro variazione (es.: la co-variazione dei componenti muscolari, tattili, di orientamento, ecc. che partecipano alla condotta del prendere). Ad un livello di ulteriore differenziazione dei processi corporei (livello 3), troviamo i comportamenti, intesi come copioni procedurali regolati in ragione del risultato cui tendono (es.: indicare, lanciare, sbucciare, aprire, guidare). Questi copioni sono cluster di cluster di co-variazione dei processi neurofisiologici di base; in altri termini, ciascun comportamento è costituito da una combinazione coordinata di condotte (es.: aprire una porta richiede la combinazione coordinata di mantenere e spostare). Una volta appresa e ripetuta sistematicamente, la combinazione diventa un'abitudine, un automatismo; tuttavia, essa mantiene un certo grado di flessibilità nel modo con cui le proprie componenti si coordinano reciprocamente, così che il comportamento possa essere modulato in ragione delle contingenze ambientali. Ad un livello ulteriore (livello 4), sono collocabili 
gli atti, intesi come combinazioni di comportamenti regolati, posti al servizio di fini definiti nell'interazione con il contesto fisico e sociale. Ad esempio, aprire la porta e salutare sono azioni che si combinano nell'atto dell'accogliere l'ospite. A questo livello la variabilità delle combinazioni è più elevata: lo stesso cluster di stati senso-motori che sostanzia una condotta o un'azione può trovarsi a concorrere alla costituzione di azioni anche tra loro molto diverse e perfino di opposto senso (es.: si afferra la maniglia della porta sia per aprire che chiudere la porta di casa; si apre la porta di casa sia come segno per accogliere l'ospite che per cacciarlo).

Un punto centrale è la struttura solo parzialmente annidata che lega i livelli descritti sopra. In altri termini, il corpo funziona in parte come una matrioska ed in parte come un contenitore disordinato di stati: alcune condotte (livello 2) sono caratterizzate in modo prevalente o quasi esclusivo da stati corporei che appartengono ad un unico (o ad una combinazione) di stati affettivi (livello 1). Tali stati di ordine logico superiore sono dunque annidati entro gli stati di livello inferiore. Ad esempio, sorridere, accarezzare, baciare sono condotte fondate su stati senso-motori che in grande prevalenza sono condivisi con lo stato affettivo piacevole; sono dunque una sottocategoria del livello 1, in essa annidata. Altri atti sono invece trasversali: "pescano" in più di una polarità affettiva. Ad esempio, l'afferrare è sostanziato da un insieme di componenti senso-motorie che non sono caratterizzati affettivamente, vale a dire non comprendono in modo sistematico le co-variazioni senso-motorie che costituiscono un determinato stato affettivo; ciò accade perché l'afferrare è un cluster di stati sensomotori limitato che può attivarsi tanto in co-occorrenza con elementi di una polarità affettiva che dell'altra (la persona afferra sia se è in condizione affettiva di piacevolezza che di spiacevolezza).

L'organizzazione trasversale degli stati aumenta con il livello di organizzazione, in quanto ai livelli di organizzazione più differenziati giocano un ruolo maggiore i segni secondari, la cui distribuzione è determinata dalle contingenze storico-culturali. Da un punto di vista complementare, come già osservato, $\mathrm{i}$ livelli superiori di organizzazione riguardano azioni ed atti che si instanziano attraverso combinazioni flessibili di condotte; di conseguenza, un certo atto può essere realizzato nei termini di stati corporei che combinano comportamenti espressione di stati corporei che appartengono a stati affettivi omogenei e/o che sono trasversali ad essi. Ciò dà ragione del fatto che mentre alcuni atti mantengono una valenza affettiva definita (es.: colpire qualcuno con un pugno al volto è per molte persone un atto compatibile solo con alcuni stati affettivi), altri sono trasversali, dunque autonomi rispetto allo stato affettivo del momento, vale a dire rispetto alla parallela configurazione che il corpo mantiene al livello 1.

È rilevante osservare come il grado di trasversalità/annidamento dei livelli di organizzazione varia tanto tra gli individui che tra i contesti socioculturali. 
A livello individuale, l'organizzazione dipende in larga misura dalla biografia del soggetto, dunque dall'esperienza di come i segni hanno strutturato la covariazione degli stati corporei; lo stesso atto potrà dunque essere annidato entro uno stato affettivo (dunque dipendente da e vincolato ad esso) per un soggetto, ed essere autonomo per un altro. La capacità di certe persone di compiere attisia positivi che negativi - anche in assenza degli stati di attivazione affettiva generalmente associati ad essi (e.g. uccidere a sangue freddo, perdonare il proprio aggressore) sono esempi di come l'organizzazione dei livelli vari tra le persone. A livello socio-culturale si può osservare come in certi contesti culturali certi comportamenti ed atti siano segregati entro una specifica configurazione affettiva, mentre in altri sono autonomi o addirittura appartengono ad una configurazione affettiva opposta (ad esempio in Italia dare una pacca sulla spalla ad un conoscente è un segno compatibile con uno stato affettivo di piacevolezza; in Finlandia sarebbe vissuto come un'aggressione; incrociare gli occhi dell'interlocutore in alcune culture è un automatismo privo di connotazione affettiva; in altre è un gesto aggressivo). Per inciso, quanto detto offre una chiave di interpretazione della funzione di elaborazione culturale dei luoghi/processi transizionali dedicati ad esplorare combinazioni inedite tra i segni - nell'umorismo, nel gioco, nello sport, nell'arte, nei luoghi di confine, alcuni atti in genere associati a stati affettivi definiti sono agiti entro contesti affettivi differenti o comunque esercitati in autonomia rispetto ai loro stati affettivi canonici. Si potrebbe dire che questi processi sono l'equivalente sociale dell'attività onirica individuale.

L'organizzazione degli stati corporei delineata sopra dà ragione del carattere multidimensionale del valore del segno. Ogni segno (lo stato del corpo cui esso corrisponde) entra in relazione con una pluralità di altri stati corporei, distribuiti su tutti i livelli di organizzazione - ad es. il suono della voce di una persona cara puo essere associato alla piacevolezza sperimentata in molti dei momenti di interazione con lei (livello 1), così come ai movimenti del braccio usati per salutarla (livello 2); in altre occasioni il suono può essere stato associato all'azione, ad esempio, di porgere qualcosa (livello 3), di scusarsi (livello 4) e così via. Il suono della voce può operare come guida dell'inferenza bayesiana su tutti questi livelli di organizzazione; il suo valore è dunque un vettore multidimensionale - sarà dato dalla distribuzione dei valori sulle diverse dimensioni. D'altra parte, tali valori saranno differenti in ragione del livello di associazione del segno con i pattern senso-motori relativi ai diversi livelli di organizzazione. Per rimanere nell'esempio proposto sopra, nella misura in cui il suono della voce è risultato nel tempo co-occorrere maggiormente con la piacevolezza dei momenti di relazione rispetto all'atto di scusarsi o salutare (in quanto questi ultimi occorrono molte volte senza che sia compresente il suono), 
tale segno tenderà ad elicitare il pattern affettivo, dunque ad instanziare il suo valore in quanto segno che attiva il livello 1 di organizzazione corporea.

\section{Organizzazione degli stati corporei e valore semiotico dei segni}

La discussione sviluppata sopra porta a concludere che la capacità discriminante del segno non dipende dalle caratteristiche intrinseche del segno stesso ma dal modo con cui i segni si distribuiscono entro le pratiche sociali. E questo è un diverso modo per affermare la concezione pragmatica del segno secondo cui il suo significato sta nel modo con cui è utilizzato (Wittgenstein, 1953/1958); infatti, il modo di utilizzare un segno altro non è che la definizione di una determinata contingenza delle co-occorrenze con gli altri segni: ogni volta che una determinata pratica sociale (intesa in senso lato) viene esercitata, il cluster di co-occorrenze di segni in cui essa consiste aumenta la propria frequenza, ridisegnando in tal modo il valore di tali segni, vale a dire la possibilità che ciascuno di essi operi da interpretante degli altri (i.e. guidi l'inferenza bayesiana).

Va tuttavia osservato che il valore non dipende solo dalla frequenza della co-occorrenza tra il segno e gli altri segni che costituiscono tale pratica sociale. Dipende anche, ed in misura essenziale, dal grado di differenziazione/omogeneità tra le pratiche sociali ${ }^{13}$. In breve, il valore di un segno dipende dalla complessiva distribuzione delle relazioni tra i segni, dunque dalla loro distribuzione entro e tra le pratiche sociali: quanto maggiore è la tendenza dei segni a specializzarsi in configurazioni tra loro differenziate, tanto maggiore, a parità di altre condizioni, sarà la capacità dei segni di stare per gli altri segni con cui entrano in rapporto.

\section{La funzione di stabilizzazione semiotica degli affetti}

La discussione proposta sopra pone le premesse per comprendere in chiave

\footnotetext{
${ }^{13}$ Si pensi, ad esempio, al segno $\alpha$ che, tutte le volte che compare, è seguito dal segno $\mu$. A tal fine, si assuma che il segno $\alpha$ sia storicamente occorso 10 volte e che dunque la co-occorrenza $\alpha \& \mu$ abbia frequenza 10 . Si immagini ora che il segno $\eta$ abbia una frequenza 1000 e che sia seguito dal segno $\mu$ nel $20 \%$ dei casi. In questo caso la co-occorrenza $\eta \& \mu$ avrà frequenza 200. Nella misura in cui il valore semiotico dipende dalla frequenza relativa della co-occorrenza, allora il segno $\alpha$ avrà un valore discriminante 5 volte superiore (100\% vs $20 \%$ ) a quello di $\eta$ (ovviamente, in rapporto $a \mu$ ), anche se il segno $\alpha$ è associato 20 volte in meno al segno $\mu$ (in termini assoluti) di quanto lo sia il segno $\eta$.
} 
computazionale il meccanismo cognitivo alla base del rapporto tra incertezza ed attivazione, riconosciuto e studiato sul piano descrittivo e funzionale dalle teorie psicosociali richiamate in precedenza. In termini generali, la tesi che si propone è che l'attivazione affettiva è il modo con cui il sistema cognitivo ricerca il fit dell'inferenza in condizione di contrazione locale del valore semiotico. Precisiamo di seguito tale tesi.

In primo luogo, dal punto di vista del sistema cognitivo l'incertezza è la condizione di perdita della capacità di fit. Questa idea è coerente con le teorie che concepiscono l'incertezza in termini di destabilizzazione del sistema di significato (e.g. Proulx \& Inzlicht, 2012). Tuttavia, a differenza di tali teorie, la tesi che in questa sede si propone considera la destabilizzazione in termini micro-analitici: come induttore di perdita di valore semiotico, vale a dire di riduzione (momentanea e relativa al processo cognitivo in corso) della capacità del segno di determinare il fit. Il segno elicita la previsione circa il segno successivo; ciò sulla base della distribuzione di co-occorrenze definitoria della sua funzione di relazione. Laddove per un motivo o per l'altro l'ambiente determina condizioni che inficiano la previsione, allora il sistema si attiva immediatamente per recuperare il fit.

Il punto centrale è che l'attivazione affettiva è il modo più efficace di recuperare il fit. Il carattere bipolare e globale dell'organizzazione degli stati corporei che sostanziano l'attivazione affettiva rende massimamente probabile l'inferenza previsionale - la previsione dovrà limitarsi a definire lo stato corporeo inferito o come persistenza dello stato corporeo attuale (cioe, come conservazione dello stato affettivo) o, laddove la previsione dovesse risultare non confermata, come spostamento sullo stato affettivo speculare (ad esempio come transizione dal piacevole allo spiacevole). In definitiva, la natura bipolare degli affetti permette di raggiungere sempre e comunque il fit, in quanto la disconferma della previsione opera ipso facto come interpretante dello stato affettivo speculare $^{14}$. Per dirla con un'immagine, a livello di organizzazione degli affetti, il sistema cognitivo cade sempre in piedi.

${ }^{14}$ Ritorna qui un aspetto messo in luce dalla psicoanalista Melanie Klein (1967): per l'infante (ma il discorso vale per i processi mentali delle persone di tutte le età), l'assenza della madre è di per sé l'esperienza della presenza della madre cattiva - nei termini qui utilizzati: il segno che alimenta l'inferenza della presenza della madre (ad esempio lo stato di fame) non trova corrispondenza nel segno successivo (la madre non è presente nel campo visivo del bambino e dunque non viene da questi percepita); tuttavia tale non corrispondenza non si traduce in una perdita del valore del segno, in quanto è ipso facto semiotizzata affettivamente: come presenza della madre cattiva - il senso di fame attiverà altri stati affettivi di spiacevolezza, in tal modo recuperando la capacità di fit. 


\section{L'affettivizzazione delle società. Una chiave interpretativa}

La discussione fin qui sviluppata offre una chiave di lettura utile a comprendere il meccanismo psicosociale sotteso all'affettivizzazione della società contemporanea. La tesi che si propone è che lo scenario socioculturale contemporaneo abbia determinato un radicale decadimento del valore dei segni, come effetto della loro progressiva de-specializzazione, a sua volta riflesso della "fuzzificazione" degli ambiti di attività in cui la vita sociale e istituzionale si articola (Salvatore, Mannarini et al., 2019). Presentiamo di seguito i punti su cui si articola tale tesi.

\section{L'iper-connettività delle società contemporanee}

In un precedente lavoro, uno di noi, con altri, ha proposto una chiave di lettura del processo cui in questa sede si riferisce il termine "de-specializzazione" dei segni (Salvatore, Mannarini et al., 2019). Un elemento centrale evidenziato da tale ipotesi interpretativa è l'iper-connettività che caratterizza le società contemporanee, come effetto delle dinamiche della globalizzazione.

Con iperconnettività si intende l'aumento esponenziale delle relazioni tra $\mathrm{i}$ vari punti dello spazio sociale, così che ogni ambito di vita si ritrova ad operare come un nodo di una rete tendenzialmente tutti-a-tutti che si estende sull'insieme degli altri ambiti/processi sociali. Ogni aspetto della vita delle persone e delle istituzioni è, almeno potenzialmente, collegato con/esposto ad un'infinita pluralità di altri elementi. Le interazioni tra i nodi della rete sono di diversa forma: funzionali (es.: l'impatto globale delle produzioni inquinanti su aree geograficamente anche molto distanti), economiche (si pensi a come le decisioni prese da alcuni istituti finanziari americani abbiano generato una crisi finanziaria globale che ha avuto enormi ripercussioni sulla vita di moltitudini sparse in tutto il mondo); politico-istituzionali (in ambiti come la protezione della privacy, la difesa del consumatore, la tutela dei diritti civili, la regolazione dell'uso di OGM, il diritto d'autore - le norme definite dalle istituzioni ed agenzie sovrannazionali incidono profondamente sulla vita quotidiana delle persone); comunicative (es.: i social media permettono alle persone di entrare ed espandere la platea degli interlocutori, sia dal punto di vista volumetrico che di estensione spaziale, che di intensificazione temporale - possiamo comunicare con sempre più persone, dislocate in qualsiasi parte del mondo, nei termini di scambi comunicativi sempre più rapidi e frequenti). 
Il costante potenziamento e dinamicizzazione delle connessioni reticolari riflette lo sviluppo esponenziale della capacità tecnologica di asseverare l'ambiente all'azione umana. L'ottimizzazione tecnologica offre sempre nuove capacità di manipolazione dei nessi di causa-effetto, di superamento dei limiti spazio-temporali e cognitivo-computazionali - nell'elaborazione delle informazioni, di utilizzazione delle risorse ambientali, di accelerazione dei processi produttivi e sociali, di incremento dell'estensione e intensità dell'impatto delle azioni umane.

\section{La fuzzificazione dei confini}

Le crescenti capacità tecnologiche stanno rendendo il mondo sempre più un'unità globale: una singola rete bio-politica ad alta densità. Di conseguenza, i confini all'interno e tra le società sono sempre più deboli, sempre più instabili, liquidi (es.: Bauman \& Bordoni, 2014); perciò, forme di vita, linguaggi, pratiche sociali, gruppi, individui sono sempre più esposti ad un'alterità generalizzata. Con il termine fuzzificazione si vuole qui intendere tale processo: la progressiva perdita di confini netti, trasformati in zone intermedie dove, come accade negli insiemi fuzzy, le condizioni alternative coesistono.

Di seguito una lista di esempi del processo di fuzzificazione della società contemporanea:

- La logica del servizio è il nuovo paradigma di business management, reso possibile dallo sviluppo di tecnologie intelligenti e dai sistemi informatici a supporto della progettazione - basata sull'idea che il consumatore è parte del processo di produzione: non è solo il fruitore del prodotto finito, ma è un pro-sumatore - ovverossia è produttore e consumatore allo stesso tempo (Norman, 1981).

- L'impressionante evoluzione dello sviluppo scientifico e tecnico in medicina ha reso più sfumata la differenza tra salute e malattia - i pazienti cronici sono in qualche modo sia malati che sani: la malattia non è qualcosa di differente dalla salute, ma una componente della stessa, e vice versa. Da una prospettiva complementare, la nuova cultura dell'assistenza sanitaria ha introdotto l'idea che la salute è una componente che va perseguita anche in condizioni di malattia (Bertini, 2012).

- La complessità di molte questioni politiche in aree come l'educazione, la salute, la sicurezza e le migrazioni rende il ruolo delle istituzioni poste a differenti livelli decisionali (transnazionale, nazionale, regionale e locale) sempre più interdipendente, senza confini chiari tra le competenze e le responsabilità proprie dei diversi livelli. 
- Le persone salvate grazie alla donazione degli organi sono esempi viventi di come la fuzzificazione dei confini possa investire anche la sfera biologica;

- La lotta per i diritti civili e il riconoscimento delle persone LGBT è un ulteriore esempio dell'obsolescenza di una segmentazione discreta del continuum bio-sociale concernente l'identità sessuale e della opportunitàdi considerare quest'ultima come un continuum qualificato da confini sfocati e mobili.

- Nella sfera politica, l'offerta populista non si presta ad essere rappresentata nei termini delle dimensioni classiche di sinistra e destra (es.: Mudde, 2004): il discorso populista fonde temi, linguaggi e proposte che storicamente sono state appannaggio tanto di tradizioni di destra che di sinistra.

- I flussi migratori stanno modificando in profondità i paesaggi urbani: forme di vita anche molto diverse tra loro (in termini di modi del comportamento, uso del tempo, abitudini alimentari, negozi, odori, colori e così via) coesistono l'uno accanto all'altro; persino dove non interagiscono direttamente tra di loro, la loro parallela esistenza non può non produrre reciproche contaminazioni; il fatto stesso che migranti di seconda (o successiva) generazione abbiano acquisito la cittadinanza del Paese dove risiedono, testimonia che la vicinanza spaziale è un motore propulsivo di contaminazione antropologica e, più in generale, è il segno dell'inevitabile obsolescenza della visione dell'identità nazionale basata sull'etnia;

- La pluralizzazione delle fonti di conoscenza e dei dati rende sempre più difficile definire una netta distinzione tra il vero e il falso, come ben compreso da coloro che creano, diffondo e traggono vantaggio dalle fake news.

Da un punto di vista complementare, è importante considerare che la tecnologia contemporanea (ad esempio l'intelligenza artificiale, la robotica, i sistemi intelligenti, le infrastrutture per la gestione dei flussi di big data) è caratterizzata da una crescente versatilità e velocità. I dispositivi tecnologici non si limitano a eseguire singole operazioni; piuttosto, essi sono sempre più in grado di gestire processi complessi impostando e finalizzando catene di input ed output caratterizzate da estrema rapidità e opacità. Ciò significa che in un numero sempre maggiore di domini di vita i processi mediati dai dispositivi tecnologici funzionano in modo sempre più autonomo e incapsulato, con il ruolo degli esseri umani relegato al controllo post-hoc dell'output del processo automatizzato ${ }^{15}$.

\footnotetext{
${ }^{15}$ Un esempio emblematico di questa tendenza è il modo in cui la tecnologia ha modificato il funzionamento del mercato azionario. Fino ad un non lontano passato, i dispositivi tecnologici fungevano da supporto per l'attività umana, ad esempio come potenziamento della capacità di analisi e di archiviazione dei dati e come modalità di riduzione dei tempi della comunicazione. Adesso, invece, essi tendono largamente a sostituirla: le decisioni sono prese direttamente da un software sulla base degli algoritmi che puntano alla massimizzazione della funzione di utilità perseguita. Il decisore interviene a monte, nella determinazione dei parametri dell'algoritmo e a
} 
Di conseguenza, il mondo globalizzato appare come un ambiente in cui i processi hanno raggiunto un livello di complessità e opacità tali da essere ormai difficilmente rappresentabili. In uno scenario di complessità di tal tipo, non solo le persone comuni, ma anche gli esperti spesso non sono in grado di comprendere il perché, il come e a volte anche il quando di ciò che accade, vale a dire di costruire e aggiornare le mappature cognitive dei fenomeni che li vedono coinvolti.

È opportuno porre l'attenzione sul fatto che l'iper-connettività e l'opacità sono due facce della stessa medaglia e si rinforzano l'una con l'altra. Con il termine iper-connettività stiamo in questa sede a significare che la logica del processo non è localizzata nell'ambiente circoscritto di un singolo (o gruppo di) nodi della rete, perciò non è rappresentabile con le risorse cognitive a disposizione del nodo. Perciò, l'iper-connettività implica di per sé l'opacità. Dall'altro canto, l'opacità riduce la capacità cognitiva del nodo di "difendere" i propri confini, vale a dire l'autonomia del proprio ambiente locale, contribuendo, in questo modo, al suo asservimento alle dinamiche globali. Si pensi a dinamiche quali la finanziarizzazione dell'economia, la deterritorializzazione del potere politico, la costruzione e diffusione delle conoscenze: tali dinamiche non consistono di un determinato gruppo - più o meno ampio - di attori portatori di specifiche scelte e comportamenti; al contrario, sono fenomeni emergenti dall'interazione latente di una rete pressoché infinita di interazioni ricorsive. Ciò significa che le categorie che le persone e i gruppi sociali utilizzano per rappresentare i comportamenti e le scelte locali non sono in grado di "afferrare" la dinamica che si realizza al livello sistemico. D'altra parte, la difficoltà a rappresentare tali dinamiche impedisce la definizione di una progettualità sociale, politica e istituzionale relativamente a tali dinamiche, favorendo la loro tendenza all'autoreferenzialità e la loro capacità a sfuggire al controllo sociale e istituzionale.

\section{Il decadimento del valore semiotico}

Dalla discussione proposta nei precedenti paragrafi discende che il valore semiotico dei segni dipende dal livello di differenziazione del sistema sociale. Quanto più il sistema sociale è strutturato, vale a dire segmentato in ambiti di

valle, controllando l'output del ciclo; tuttavia, il processo in sé è molto veloce e in gran parte autonomo - la cronaca ha registrato svariati episodi di effetti a catena dovuti a falle del software, che hanno comportato perdite di centinaia di milioni di dollari, prima che l'intervento umano fosse capace di recuperare il controllo del processo. 
attività stabili nei loro rapporti e nel tempo, con ruoli, norme, confini sufficientemente delineati, tanto più la combinazione di segni secondari che caratterizza ciascun ambito sarà specifica di quell'ambito; al contrario, quanto maggiore è il livello di differenziazione del sistema sociale - vale a dire, la tendenza degli ambiti a perdere confini netti ed a contaminarsi reciprocamente quanto a ruoli, modelli di pensiero e di azione - tanto meno le combinazioni di segni avranno specificità, potendosi gli stessi segni combinare in una pluralità di modi alternativi; in conseguenza di ciò, i segni perderanno capacità di discriminare, dunque valore semiotico.

Si può comprendere come la fuzzificazione della società contemporanea, unita alla opacità di tale dinamica (Salvatore, Mannarini et al., 2018), determini, sul piano dei processi socio-cognitivi, la caduta del valore semiotico dei segni. $\mathrm{Da}$ un lato, culture, modelli di comportamento, valori si trovano sottoposti a spinte omogeneizzanti; dall'altro, la difficoltà a rappresentarsi i processi in cui sono immersi rende difficile alle persone individuare cornici interpretative che mettano in qualche modo ordine, antagonizzando la pressione omogeneizzante.

Per inciso, tale tesi interpretativa fornisce una chiave di lettura per comprendere perché l'evoluzione di alcuni comportamenti sociali - ad esempio, nella sfera degli orientamenti di genere, il matrimonio tra persone dello stesso sesso o l'assunzione di ruoli tipicamente femminili da parte di uomini - tendano ad innescare forti reazioni emotive in molte persone, come se si trattasse di fatti catastrofici. Chi reagisce in tal modo non lo fa perché si sente direttamente danneggiato; piuttosto, attraverso tali risposte affettive cerca di fronteggiare il "terremoto semiotico" scatenato dalla fuzzificazione dello spazio sociale veicolata da tali cambiamenti - un ordine sociale, e dunque semiotico, è andato perduto e gli affetti si offrono come suo surrogato.

\section{Per concludere. L'affettivizzazione è una strategia cognitiva}

Quanto discusso fin qui porta ad una conclusione generale. L'affettivizzazione della sfera pubblica non è una mera reazione emotiva alla destabilizzazione di modi di vivere consolidati indotta dalla globalizzazione. L'affettivizzazione è il modo che il sistema cognitivo delle persone adotta per dare un senso ai nuovi scenari dell'esperienza; in altri termini, non è sintomo del fatto che il sistema cognitivo abbia smesso di funzionare e così facendo abbia lasciato spazio all'irrazionalità; piuttosto, è la fondamentale strategia adottata dal sistema cognitivo in situazione di incertezza. Gli occhiali con lenti nere non si usano per diventare ciechi ma per vedere al meglio in condizioni difficili che sfidano il sistema ottico. 
Un indicatore esemplificativo di come questo processo sia onnipresente e ci coinvolga tutti, può essere individuato nel modo con cui sono riportate le notizie di incidenti (es.: esplosioni, aggressioni, incendi) nei notiziari. Tendenzialmente, le prime informazioni fornite riguardano se si sia trattato di un attacco terroristico o meno; questo aspetto sembra dunque più rilevante dell'entità dei danni, della eventuale presenza di vittime e dell'organizzazione dei soccorsi. Tuttavia, sarebbe sbagliato considerare tale priorità una forma di irrazionalità (o una forma di manipolazione finalizzata ad assecondare l'attivazione affettiva del pubblico); piuttosto, è il modo in cui la cognizione gestisce l'incertezza generata dall'evento: il sistema cognitivo attiva lo schema affettivo amico-nemico che rende pertinente dell'evento la sua qualità di essere un "attacco terroristico" vs non esserlo. In tal modo, il segno "non è un attacco terroristico" è caricato di valore semiotico, ovvero diventa un vettore di informazione (capace di sollecitare una reazione emotiva di sollievo, come se si fosse in presenza di una buona notizia). Ciò avviene indipendentemente dal fatto che, dal punto di vista logico, affermare una negazione ("non è un atto terroristico") di per sè non produce informazione. In realtà tuttavia, dal punto di vista degli affetti, la negazione di uno stato è l'affermazione del suo opposto.

\section{Come contrastare l'affettivizzazione. Un'osservazione generale}

Le manifestazioni critiche dell'affettivizzazione della sfera pubblica portano a valutare il contrasto della stessa come un obiettivo desiderabile, un modo attraverso il quale contribuire al progresso sociale, ad esempio in termini di difesa delle istituzioni democratiche, promozione del senso civico, riduzione della polarizzazione sociale e ideale, argine al razzismo, alla xenofobia e così via. Ma come è possibile operare in tal senso?

\section{Riduzione dell'incertezza e capacità di affrontarla semioticamente}

Il modello della relazione che intercorre tra incertezza e attivazione affettiva discusso in precedenza (cfr. $\S 3-5$ ), unitamente al riconoscimento del ruolo di mediazione giocato dal valore semiotico, forniscono una possibile risposta a questo interrogativo. Da una parte, appare ovvio che i generatori di incertezza (diseguaglianza, finanziarizzazione dell'economia, radicalizzazione dei conflitti geo-politici, crisi ambientale) debbano essere indeboliti, così da poter ridurre il bisogno di ricorrere all'affettivizzazione per proteggere i segni del decadimento del loro valore semiotico (cfr. $\S 4.1$ e 5.2). D'altra parte, devono 
essere sviluppate risorse semiotiche innovative (Salvatore, Mannarini et al., 2018), utili a potenziare le capacità dei soggetti di ridurre l'impatto destabilizzante che l'incertezza esercita sul sistema cognitivo.

Quest'ultima strategia ha senso in quanto l'incertezza riguarda la relazione epistemica tra il soggetto e il mondo. In altre parole, l'incertezza non è una caratteristica ontologica, inerente al mondo; ma è piuttosto il complemento della capacità del sistema di significato messo in gioco per mappare l'ambiente in funzione delle richieste dell'azione (cfr. § 3.3). Un esempio può aiutare ad evidenziare questo rilevante passaggio. Si immagini un pubblico ufficiale che presiede una cerimonia istituzionale regolata da norme rigidamente formali; ad un certo punto, improvvisamente, l'ufficiale inizia a manifestare dei comportamenti inaspettati, in contrasto con il suo ruolo e la rigida etichetta - ad esempio salta sul tavolo, inizia a ballare e, spogliandosi, urla dell'ormai imminente fine del mondo. I partecipanti alla cerimonia, a bocca aperta, iniziano a pensare che sia ubriaco o che sia vittima di un episodio psicotico. Questa vignetta mostra come l'incertezza possa essere affrontata attraverso la mobilitazione di segni innovativi piuttosto che attraverso il mero ricorso all'attivazione affettiva. Il comportamento bizzarro dell'ufficiale ha generato un aumento dell'incertezza: Come esito di tale rottura, segni solitamente specifici di un certo tipo di pratiche - ad esempio di pratiche quali svago/socializzazione (danzare), intimità (nudità), pratiche religiose (affermazioni escatologiche) - vengono ad essere mischiati in un unico crogiolo omogeneizzante (cfr. sopra, §4 e §5). Questo equivale a dire che tali segnali in tal modo perdono la propria specializzazione e conseguentemente il loro valore semiotico - non sono più in grado di generare inferenze efficaci circa la traiettoria dei segni successivi. Nuovi segni introdotti dai partecipanti per interpretare l'evento di rottura - ad esempio "è ubriaco", "sta diventando pazzo", - individuano un nuovo schema di relazioni sufficientemente stabili tra i segni interpretati (le persone ubriache o pazze possono essere trovate a danzare su un tavolo, senza curarsi dei limiti derivanti dall'etichetta, dalla decenza e dalla plausibilità) e così facendo ripristinano, seppure alla luce di una diversa prospettiva, la loro specializzazione.

L'astrazione è uno dei modi tramite il quale si può ripristinare il valore semiotico dei segni. Solo per fare un esempio, si pensi ad un analista alle prese con una serie ampia di valori varianti nel tempo. Se l'analista dovesse focalizzare la propria attenzione sui singoli valori, gli sarebbe impossibile identificare relazioni sufficientemente stabili, dunque sensate; la serie apparirebbe solo come rumore. Nel momento in cui si sposta ad un livello più astratto, ad esempio calcolando la media e la sua variazione lungo l'arco temporale, dal rumore emerge una tendenza riconoscibile, una relazione sovra-ordinata tra i valori. Per fare un esempio più vicino all'argomento al centro della presente discussione, si prenda il caso della categoria "studenti dotati". Prima della sua diffusione, i 
contesti di insegnamento-apprendimento che coinvolgevano studenti rientranti in tale categoria erano caratterizzati dalla disorientante compresenza di segni che ci si aspettava essere specifici di due pratiche educative tra loro opposte le pratiche riguardanti la gestione educativa di capacità cognitive elevate e le pratiche riguardanti la gestione delle difficoltà/insuccessi dell'apprendimento. Una volta che la nozione di "studente dotato" si è radicata all'interno del sistema educativo, essa ha trasformato i due tipi di segni in una configurazione sensata, ripristinando in tal modo la capacità di fit dei processi cognitivi che regolano e sostanziano quest'area dell'azione educativa ${ }^{16}$.

Mentre la riduzione dei generatori di incertezza riguarda scelte istituzionali e socioeconomiche, la promozione di risorse semiotiche innovative (ad esempio visioni, valori, categorie interpretative, rappresentazioni dell'alterità) protettive dall'incertezza è faccenda che chiama direttamente in gioco la definizione di politiche educative e socio-psicologiche di sviluppo culturale (Salvatore, Mannarini et al., 2018). Conseguentemente, la discussione che segue si concentrerà su quest'ultimo aspetto.

\section{Il radicamento socio-psicologico dei segni innovativi}

Il principale problema socio-psicologico relativo allo sviluppo di segni innovativi è come promuovere il radicamento di tali risorse nella vita istituzionale e sociale. In altre parole, si tratta di comprendere attraverso quale strategia i nuovi significati prodotti negli ambiti della cultura, delle arti, della scienza e della vita civica, possano acquisire "valore di vita" (sul concetto di valore di vita, si rimanda a Salvatore et al., 2012) - ossia la capacità di agire come regolatori viscerali (embodied) dei pensieri, sentimenti e scelte di individui e gruppi. ${ }^{17}$

\footnotetext{
${ }^{16}$ Malattia cronica e LGTB sono altri esempi di significati innovativi. Ognuno di essi riscrive la relazione tra le componenti di un certo ambito dell'esperienza, potenziando in tal modo la capacità interpretativa del soggetto interpretante. Dove prima il soggetto era esposto alla confondente compresenza di segni appartenenti a sfere diverse dell'esperienza (la malattia e la salute, la mascolinità e la femminilità), il significato innovativo gli permette di individuare una nuova forma/oggetto dell'esperienza (si rimanda anche a Gonçalves e colleghi, 2011 per una discussione clinica sul ruolo dei significati innovativi nella elaborazione narrativa dell'esperienza).

${ }^{17}$ Ad esempio, si consideri quanto sia diffusa la pirateria televisiva in tutta Europa. Milioni di persone sono clienti di fornitori illegali. Tutte queste persone sanno che si tratta di un crimine. Eppure, per molti di loro questo segno (pirateria = reato) è un significato "vuoto", privo di valore di vita, incapace di incanalare altri pensieri e sentimenti su se stessi e sull'atto di utilizzare il sistema della pirateria. Ne risulta che molte persone possano sentirsi oneste, operare in tal senso in molti ambiti di vita, e allo stesso tempo portare avanti tale pratica illegale.
} 
La risposta, proposta a seguire, a questo problema centrale è basata sul riconoscimento della situatività della cognizione. Come detto in precedenza $(\S 3.3)$, con questa espressione si intende dire che i significati sono al servizio delle azioni, e perciò immanenti alle pratiche sociali in cui consistono le azioni. Ciò significa che ogni significato acquisisce la capacità di lavorare come un regolatore cognitivo nella misura in cui e per mezzo del fatto che fonda e struttura la coordinazione della pratica sociale di cui è parte integrante (cfr. $\$ 3.3$ e $\S 3.5) .{ }^{18}$ Per dirla in breve, il significato è l'interiorizzazione dell'organizzazione dinamica della pratica sociale ${ }^{19}$. Ciò che Matteo riporta come insegnamento dato da Gesù ai suoi discepoli - "Dove due o tre sono riuniti nel mio nome, io sono in mezzo a loro" (Matteo, 18, 20) - fornisce la rappresentazione plastica di questa tesi: un significato (essere "in mezzo a loro") è dotato di valore di vita - ossia ha la capacità di rappresentare la presenza di Gesù come un fatto - nella misura in cui la messa in atto di un segno (essere "nel mio nome") motiva e configura il coordinamento dell'azione ("dove sono due o tre riuniti"). In breve, un significato è reale - ha valore di vita per il soggetto quando, e a condizione che, vivendolo fa sì che la pratica sociale fondata su di esso sia riprodotta nel corso del tempo (Salvatore, 2012, 2019). ${ }^{20}$

Una volta che viene presa in considerazione la situatività dei segni, si è portati a concludere che le persone non accomodino i propri sistemi di significato per accogliere segni innovativi solo perché qualcuno insiste affinché così si fac$\mathrm{cia}^{21}$. Ciò che è necessario per promuovere un tale accomodamento sono pratiche sociali che incapsulino i segni innovativi, vale a dire pratiche sociali che

${ }^{18}$ Ciò avviene perché la capacità di adattamento del segno che qualifica il suo valore semiotico, e di conseguenza il suo valore di vita, è stabilito nel corso dell'azione e per il bene dell'azione: la capacità di adattamento è la capacità del segno di regolare l'azione.

${ }^{19}$ È possibile trovare versioni di questo principio basilare nel lavoro di autori come Durkheim (1893/1984), Klein (1967), Vygotsky (1978), Cole (1996), per citarne alcuni.

${ }^{20} \mathrm{Il}$ valore economico del denaro fornisce un altro esempio della natura sociale del valore semiotico. Il denaro è un segno che non contiene in se stesso il proprio significato (i.e. il suo valore economico). Piuttosto tale significato è l'effetto globale del fatto che la pratica sociale dello scambio economico mantiene la propria coordinazione attraverso la messa in atto del proprio presupposto (cioè l'assunto che la moneta abbia valore economico). È l'esercizio della pratica sociale che alimenta la transizione di segni (ossia, scambi, risparmi, scommesse, etc.) a generare il significato dei segni, piuttosto che il contrario; finché un nuovo segno riprodurrà tale coordinazione - nello specifico, finché un'ulteriore transazione economica/finanziaria occorrerà - il significato del denaro (ossia il suo contenuto economico, piuttosto che quello estetico e materiale) avrà valore di vita per coloro che prendono parte a tale pratica.

${ }^{21}$ Più precisamente, l'inefficacia di strategie di promozione dei segni innovativi basate sulla loro affermazione esplicita è una funzione del livello di generalizzazione del segno innovativo. Infatti, più l'innovazione semiotica è generalizzata, più profondo sarà l'impatto che avrà sul sistema di significati del soggetto interpretante; di conseguenza maggiore sarà lo sforzo di riorganizzazione delle assunzioni di base richiesto per poterlo accogliere. Apprendere che Dodoma è la capitale della Tanzania non richiede alcuna riorganizzazione del sistema di significato in colui 
abbiano tali segni incorporati nel proprio modo di operare, nella loro organizzazione. Riassumendo, se si vuole radicare significati innovativi nella cultura di un gruppo umano, tali significati (credenze, valori, principi) non vanno resi oggetto di appelli/invocazioni di principio; è necessario piuttosto progettare pratiche sociali che abbiano tali significati come loro nucleo immanente, fondativo e regolatore.

\section{Il setting terziario}

Avanzando nella discussione relativamete alla promozione di significati innovativi, introduciamo di seguito la distinzione tra tre tipi di pratiche sociali: setting primari, secondari e terziari.

I setting primari sono quegli scambi sociali nei quali i fini coincidono con la messa in atto dello scambio stesso. Di conseguenza, in questo tipo di pratiche l'esperienza di partecipazione allo scambio satura completamente il loro significato, quindi il modo di regolarlo. Il chiacchierare tra amici è l'esempio emblematico di setting primario: un legame che è messo in atto con il fine di essere sperimentato in sé, attraverso la sua riproduzione.

I setting secondari sono quegli scambi sociali inscritti (ossia limitati, finalizzati) da una regola espressione della salienza di un'istanza esterna rispetto alla pratica stessa. In altre parole, il setting secondario incorpora una mappa normativa della relazione con il mondo. Il perseguimento di uno scopo è un esempio prototipico di setting secondario. In tale setting la rappresentazione di uno stato desiderato della relazione tra pratica sociale e mondo (i.e. lo scopo) opera da cornice normativa regolativa. Il significato del setting secondario, dunque, non consiste (solo) nell'esperienza dello scambio, ma nella sua relazione di coerenza (intesa in senso lato) con la struttura esterna. Le attività produttive sono il prototipo dei setting secondari (Carli \& Paniccia, 1999; Carli \& Salvatore, 2001): in questo tipo di pratiche, gli attori non si relazionano gli uni con gli altri in ragione del legame in sé; ma per perseguire un obiettivo, altro ed ulteriore rispetto al legame; di conseguenza, il loro rapporto è regolato e limitato da - e fonda il proprio significato su - la funzione che persegue.

Il setting terziario è una sottocategoria del setting secondario. Come il setting secondario, esso è regolato da un'istanza esterna. Ciò che differenzia il

che apprende; piuttosto è il metodo attraverso il quale il sistema di significato si riproduce. Conseguentemente, è plausibile attendersi che questa innovazione sarà accolta da chi è esposto ad essa. Realizzare che apparteniamo alla stessa umanità universale degli abitanti di Dodoma può, per alcuni, equivalere ad un cambiamento radicale delle credenze fondamentali alla base del proprio senso di identità. Di conseguenza, è plausibile che l'assimilazione di simile significato sia tutt'altro che piano per queste persone. 
setting terziario dal setting secondario è la caratteristica della struttura esterna - abbastanza stabile e invariante nel tempo e nelle circostanze nel caso del setting secondario, dinamica e variabile nel caso del setting terziario. La conseguenza principale della dinamicità della struttura del setting terziario è il fatto che il sistema di segni che mediano e regolano questo tipo di pratiche (e.g., codici comunicativi, schemi interpretativi, modelli organizzativi, copioni, credenze) deve essere frequentemente aggiornato, così da poterlo mantenere accoppiato con l'ambiente. Alla luce di ciò, proponiamo di indicare la terzietà come quel tipo di configurazione ambientale che indebolisce la capacità interpretativa/regolatoria del segno, e che facendo ciò opera da richiesta per lo sviluppo/aggiornamento della stessa. In altre parole, la terzietà è la condizione che impedisce alla pratica sociale di saturare - ossia di istituire come stato di fatto dato per scontato - il sistema di significatooperante. L'estraneo è il modello della relazione con la terzietà (ad esempio, la relazione implicata in pratiche quali ospitalità, integrazione, esplorazione dell'ignoto) e fornisce l'immagine prototipica del setting terziario. L'estraneo è l'altro, il non-amico; tuttavia, l'estraneo è anche il non-nemico (Paniccia, 2003). Pertanto, la relazione con l'estraneo è la condizione epistemica in cui lo stato semiotico |non-amico| non instanzia la polarità speculare essere-nemico; rimane piuttosto come uno stato di equilibrio instabile, una condizione liminale (Stenner \& Moreno_Gabriel, 2013: vedi anche Salvatore \& Venuleo, 2017) di sospensione del significato dato per scontato (ovvero lo schema amico/nemico). Tale stato di equilibrio instabile è lo spazio semiotico dove è possibile lo sviluppo di interpretazioni innovative (vedi anche Montesarchio, \& Crotti, 1993) ${ }^{22}$. La caratteristica principale del pensiero scientifico - perlomeno dal punto di vista ideale - consiste

\footnotetext{
${ }^{22}$ Nei termini della teoria kleiniana (cfr. nota n. 14), l'estraneo è la sospensione della affettivizzazione dell'assenza - più precisamente, del fatto che l'assenza della madre buona è ipso facto la presenza della madre cattiva. Sempre da una prospettiva psicoanalitica, come descritto dal modello Edipico, la relazione con la terzietà può essere concettualizzata come riconoscimento luttuoso, ma generativo, dell'oggetto dell'oggetto del desiderio, nella sua duplice valenza. Da un lato, l'oggetto dell'oggetto del desiderio è il limite alla tendenza all'assolutizzazione del desiderio del soggetto - precisamente, dell'idea di avere l'oggetto solo per sé e di essere l'unico e solo oggetto dell'oggetto del desiderio (ad esempio, il bambino richiede che la madre si dedichi a lei/lui in ogni momento e in modo esclusivo, come se fosse il suo unico interesse); allo stesso modo, la presenza del terzo (ad esempio il marito/padre) è il limite frustrante del desiderio del bambino. D'altra parte, l'oggetto dell'oggetto del desiderio è la linfa vitale dell'oggetto desiderato (l'oggetto dell'oggetto è l'interesse, la passione, la curiosità, le aspettative, le emozioni che alimentano la vita dell'oggetto); questo è il motivo per cui Winnicott (1952/1975) considera salutare la madre "sufficientemente buona", piuttosto che la madre completamente buona quest'ultima sarebbe un contenitore a totale disposizione del bambino, tuttavia per questo motivo vuoto. In altri termini, in ultima istanza, desiderare un oggetto significa desiderare l'oggetto dell'oggetto desiderato; di conseguenza, l'oggetto dell'oggetto desiderato - il terzo - non è solo il limite, ma anche la fonte e la condizione del (sempre e necessariamente parziale) appagamento
} 
nell'essere un'attività ricorsiva di falsificazione e sviluppo del proprio stesso output: ogni prodotto scientifico è obsoleto e richiede di essere superato nel momento immediatamente successivo alla propria produzione. In ciò si può vedere il suo essere un'istanza di setting terziario: il significato prodotto non è né difeso come una verità definitiva né gettato via - non è né amico né nemico ci si riferisce ad esso come ad una credenza provvisoria che sollecita ulteriore innovazione.

Vale la pena in proposito evidenziare due aspetti.

In primo luogo, va sottolineato che la tipologia delle pratiche sociali non riguarda loro caratteristiche intrinseche; piuttosto, la tipologia concerne come le pratiche sociali sono interpretate dagli attori e di conseguenza come operano. Questo significa che una determinata pratica può funzionare come setting primario, secondario o terziario. Per esempio, legami familiari come anche organizzativi e istituzionali possono funzionare come primari - se sono interpretati in maniera autoreferenziale, come fini in sé - o secondari - se sono (più o meno parzialmente) asservite a strutture che li trascendono- o terziarie- nella misura in quale tali strutture siano considerate dinamiche e instabili, e dunque richiedenti la produzione ricorsiva di forme innovative di relazione con l'ambiente della pratica. ${ }^{23}$

In secondo luogo, è plausibile supporre che ci sia una relazione tra il modo in cui funzionano le pratiche sociali (setting primari, secondari e terziari) e le forme di organizzazione cognitiva che sono alla loro base (cfr. § 4.2). Più in particolare, si avanza la congettura che i setting primari siano associati con la prevalenza del livello embodied dell'organizzazione cognitiva, come risultato del fatto che tali setting si esauriscono all'interno del campo esperienziale al quale appartengono. In altre parole, un setting primario richiede processi cognitivi il cui compito è quello di regolare gli oggetti (cose e persone) che sono presenti nella situazione della pratica, in accordo con criteri che sono relativi alla relazione immediata con tali oggetti (i.e. le "affordance"). I setting secondari richiedono processi cognitivi i cui compiti comprendono la considerazione di che cosa è fuori/trascende il setting immediato - ovvero le regole/gli scopi. Questi processi cognitivi richiedono perciò un livello superiore di astrazione, necessario per rappresentare ciò che è sensorialmente assente (in quanto esterno al setting immediato). In ultimo, il setting terziario richiede riflessività (Salvatore, Marsico \& Andrisano-Ruggieri, 2015), ossia un tipo di cognizione che

del desiderio - e in ciò consiste la dialettica tragica del desiderio, ossia, nel fatto che il desiderio tende a desiderare la condizione assoluta che lo distrugge e dunque può realizzarsi solo a condizione del suo parziale inappagamento.

${ }^{23}$ Per inciso, mentre si può osservare che la condizione di anomia è caratterizzata dall'incidenza di pratiche sociali primarie, il capitale sociale può essere interpretato come la capacità che una società ha di impegnarsi in pratiche secondarie. 
pone i propri prodotti come oggetto di ulteriore elaborazione cognitiva, e nel fare ciò permette di antagonizzare la tendenza a trasformare tali prodotti in stati di fatto dati per scontati.

\section{La funzione del setting terziario per ri-generare valori semiotici}

La nostra tesi si basa sulla distinzione delineata nel paragrafo precedente. Proponiamo di considerare il setting terziario l'incubatore dei processi di creazione di senso che sono in grado di (ri)generare il valore semiotico, in una maniera differente e progressiva rispetto alle modalità semplificate dell'affettivizzazione.

Un punto rilevante associato a questa tesi riguarda il fatto che essa implica che in determinate circostanze - vale a dire, nel setting terziario - l'incertezza e la conseguente perdita di valore semiotico produce innovazione dei significati invece che affettivizzazione. Approfondire perché ciò avvenga va oltre gli obiettivi di questo lavoro. In questa sede ci limitiamo ad evidenziare che una possibile risposta vada ricercata nella peculiare struttura della pratica sociale terziaria. Infatti, nel caso delle pratiche terziarie, la perdita di valore è locale, riguarda un'area circoscritta della organizzazione generale della pratica. Ciò significa che in tale tipo di pratiche la maggior parte del valore dei segni è mantenuto, non viene scalfito dalla perturbazione, che rimane, per l'appunto, circoscritta. Conseguentemente, il sistema cognitivo non si trova nella necessità di recuperare valore semiotico tramite l'affettivizzazione.

Il gioco è un esempio della dialettica locale-globale qui proposta. Quando i giocatori interagiscono gli uni con gli altri attraverso la mediazione fornita dalle regole del gioco, stanno dando per scontate tali regole; nel fare ciò stanno producendo un drastico decadimento del valore dei segni che solitamente regola le loro interazioni sociali fuori dal setting del gioco; tuttavia, questo decadimento è locale: riguarda l'ambito limitato nel tempo e/o nello spazio della finzione ludica; al di fuori di tale ambito il valore canonico del sistema dei segni è preservato. In altre parole, le persone sospendono la realtà istituita delle loro interazioni - ossia, si comportano come se fossero personaggi del gioco; fanno tuttavia ciò solo in un momento/ambito circoscritto (i.e. durante il gioco). In ultima istanza, tale sospensione locale opera come uno dei modi della riproduzione della realtà complessiva della loro interazione. Poiché il declino del valore rimane circoscritto e funzionale alla riproduzione della pratica nel suo complesso, quest'ultima rimane sufficientemente stabile da assimilare la produzione di novità semiotica generata dal gioco; in tal modo la perturbazione locale non genera la necessità del ricorso alla semplificazione affettiva. 
In breve, secondo questo punto di vista i setting terziari sono setting secondari dotati di una struttura gerarchica che permette di circoscrivere ad evento locale il declino del valore dei segni, così che tale circostanza possa operare al servizio della innovazione piuttosto che della semplificazione semiotica.

\section{La domanda di istruzione}

\section{L'istruzione universitaria come setting terziario}

Affermiamo la tesi che le istituzioni formative possono - e dovrebbero giocare un ruolo strategico nel contrastare l'affettivizzazione della sfera pubblica. Le pratiche educative, e in particolare i contesti dell'istruzione universitaria, si prestano ad operare da incubatori di setting terziari, dunque come vettori dell'innovazione semiotica necessaria per far fronte in modo costruttivo all'incertezza radicale della contemporaneità, così da alimentare progresso civile, istituzionale e sociale.

E bene evidenziare due aspetti sottesi a tale tesi.

In primo luogo, si può affermare che l'istruzione sia per definizione un setting terziario. Infatti, l'istruzione è il processo sociale attraverso il quale la conoscenza implicita embodied prodotta all'interno del setting primario dei legami familiari, viene sottoposta ad una progressiva sospensione e/o perturbamento, allo scopo di mettere l'educando in condizione di sviluppare significati più avanzati (conoscenze, valori, credenze, atteggiamenti, forme di comportamento), in grado di potenziare la sua capacità di fronteggiare le richieste del mondo esterno. Ciò significa che le pratiche educative operano "naturalmente" come setting terziario. Dal punto di vista dell'educando, infatti, la relazione con il mondo è una condizione strutturale di terzietà: è l'esperienza dell'esposizione alla realtà della vita esterna ai legami primari, che sfida il sistema di significati costruito all'interno di tali legami. Inoltre, è utile evidenziare che le pratiche educative sono gerarchiche, nel senso che abbiamo dato al termine in precedenza: il legame educatore-educando costituisce un dominio locale della più generale relazione con il mondo. In questo dominio locale, l'educando può fare esperienza di un decadimento del valore del proprio sistema di significati, in modo circoscritto, attenuato, non catastrofico, e facendo ciò crea le condizioni per la produzione di innovazione semiotica, ovverossia di apprendimento.

In secondo luogo, l'istruzione è una pratica sociale che può essere vista come doppiamente terziaria. Infatti, oltre al fatto che, come detto sopra, determina il setting terziario come propria modalità di funzionamento, esercita (o comunque è in condizione di esercitare) la funzione di produrre le competenze 
richieste per interpretare le altre pratiche sociali costitutive nella vita sociale, istituzionale e organizzativa in termini di setting terziario. E questo è un altro modo di dire che le istituzioni educative hanno la missione strategica di farsi carico della richiesta di senso proveniente dalla contemporaneità.

\section{Istruzione universitaria tra setting secondario e terziario}

Nella parte a seguire ci focalizziamo sull'istruzione universitaria. Tale livello dell'istruzione si confronta con i processi sopra descritti in modo rilevante e specifico, secondo due prospettive.

Da un lato, l'espansione della relazione con il mondo aumenta progressivamente lungo il processo educativo, per raggiungere l'apice con l'istruzione universitaria. Ciò significa che mentre uno studente più giovane intrattiene relazioni con l'ambiente, limitato in ciò (e allo stesso tempo protetto) da costrizioni cognitive, logistiche, funzionali e sociali, uno studente universitario, assieme all'espansione temporale e spaziale della propria sfera d'azione (e.g. pianifica il futuro e i piani possono coinvolgere spostamenti geografici), è esposto alla complessità globale del mondo e alle sue fonti d'incertezza. In breve, nel caso dell'istruzione universitaria, i soggetti coinvolti (educatori e educandi) si ritrovano esposti alla condizione di terzietà del mondo senza mediazioni protettive. Dall'altro, le competenze che l'istruzione universitaria promuove nel presente sono quelle che giocheranno un ruolo centrale nel fondare e configurare le pratiche sociali del domani. Quindi, l'istruzione universitaria ha la responsabilità di produrre le risorse semiotiche necessarie perché i corpi sociali (istituzioni, reti sociali) siano in grado di costruire pratiche sociali capaci di rendere l'incertezza un attivatore di progresso civile e culturale, piuttosto che un veicolo di semplificazione emozionale della complessità del mondo contemporaneo.

C'è una crescente consapevolezza della responsabilità dell'istruzione universitaria nella promozione di progresso civile e sociale. In Europa (e.g. Council of European Union, 2018) così come in Italia (e.g. Rumiati et al., 2018). Si è compreso che la complessità degli scenari socioeconomici richiede che istituzioni educative, e tra questa l'università, promuovano nuovi tipi di competenze. In questa prospettiva, le recenti raccomandazioni del Council of European Union sono particolarmente indicative. Viene riconosciuta la dinamicità dello scenario sociale ed economico contemporaneo e la conseguente necessità di nuovi requisiti di competenza riguardo il lavoro ma più in generale la vita (i.e. Competenze personali, sociali e ad apprendere ad apprendere; Competenze di cittadinanza; Competenze alla consapevolezza culturale e espressiva). Queste competenze sono concettualizzate come la capacità di utilizzo in tempo reale 
delle conoscenze, al fine di mantenere finalizzata l'azione entro contesti in rapida evoluzione, in tal modo generative di autonomia e innovazione ${ }^{24}$.

D'altra parte, lo sviluppo di un nuovo corso nell'educazione può avvantaggiarsi di una comprensione più profonda delle condizioni di scenario sottostanti la domanda sociale e istituzionale di nuove competenze. Più specificamente, è rilevante comprendere la peculiare domanda cognitiva generata da tale scenario, ossia le risorse semiotiche necessarie ad alimentare i processi di costruzione di senso che le persone sono chiamate a realizzare nel nuovo scenario

La tesi proposta sopra è che lo scenario contemporaneo non è solo una questione di aumentata dinamicità e automazione dei processi socio-politici ed economici - come sembra essere implicito nel dibattito corrente sulle nuove competenze; andando più a fondo, lo scenario contemporaneo si caratterizza per la profonda trasformazione dell'orizzonte culturale, generativo di incertezza radicale e perdita di senso; in altri termini, lo scenario contemporaneo determina la strutturale incapacità dei sistemi cognitivi di sostenere modalità efficaci di attribuzione di senso (efficaci nel senso di essere in grado di alimentare progresso civile e sociale). È in tale caratteristica che lo scenario contemporaneo si qualifica, come detto nell'introduzione, in termini di frattura antropologica.

La differenza tra le due ipotesi interpretative - aumentata dinamicità vs perdita di senso - è sottile ma rilevante. La prima ipotesi afferma che il sistema cognitivo deve aumentare la propria appropriatezza alle contingenze ambientali in rapido cambiamento, in funzione di ciò da cui dipende il valore dell'output cognitivo (i.e. una contingenza potrebbe premiare la prontezza della prestazione cognitiva, mentre in un'altra contingenza l'accuratezza può costituire il principale parametro di valore). Ciò significa che il sistema cognitivo deve sviluppare regole/procedure/schemi di second'ordine che: a) mappino organizzazione della rappresentazione della conoscenza posseduta; b) mappino la contingenza ambientale in questione; c) impostino il formato più appropriato di conoscenza da estrarre dall'organizzazione globale. Come si può notare, questi requisiti corrispondono al potenziamento del sistema cognitivo nella direzione dello sviluppo di funzioni meta-cognitive che aumentano l'autonomia, l'autoregolazione e la sua capacità di adattamento all'ambiente. In ultima analisi, questa ipotesi implica l'idea che in contesti altamente dinamici, il sistema cognitivo non può funzionare solo in termini di procedure/algoritmi invarianti attivati da regole "se-allora" ma necessita di operare sulla base di euristiche che

24 "Nell' economia della conoscenza, la memorizzazione di fatti e procedure è importante, ma non sufficiente per conseguire progressi e successi. Abilità quali la capacità di risoluzione di problemi, il pensiero critico, la capacità di cooperare, la creatività, il pensiero computazionale, l'autoregolamentazione sono più importanti che mai nella nostra società in rapida evoluzione. Sono gli strumenti che consentono di sfruttare in tempo reale ciò che si è appreso, al fine di sviluppare nuove idee, nuove teorie, nuovi prodotti e nuove conoscenze" (p. C/189/2, punto 7). 
costruiscano rappresentazioni funzionali dello spazio del compito e regolino l'appropriatezza della risposta. E ciò equivale a dire che l'ipotesi della dinamicità prevede un sistema cognitivo che è chiamato non solo a portare al termine il proprio compito, ma anche a regolare la propria performance tramite una rappresentazione costruttiva della relazione con l'ambiente contingente da cui dipendono le condizioni della performance e il suo significato.

Ora, è evidente quanto tale visione costruttiva del sistema cognitivo sia rilevante, specialmente in ambienti dinamici; tuttavia, essa non prende in considerazione l'aspetto che è al centro dell'ipotesi della perdita di senso - ossia l'idea che oltre una certa soglia, la dinamicità dell'ambiente cambia qualitativamente e con tale cambiamento l'intero compito assume una natura differente: diventa un altro gioco - più specificamente, il gioco di come il sistema cognitivo possa affrontare la mancanza delle risorse semiotiche necessarie perché possa operare.

In breve, la principale differenza tra le due ipotesi è la seguente. L'ipotesi dell'aumentata dinamicità assume che il sistema cognitivo possieda le risorse semiotiche necessarie per operare - i.e. può basarsi su segni che mantengono il proprio valore, capaci di fornire mappe cognitive dell'ambiente interno ed esterno funzionali. L'ipotesi della perdita di senso non si focalizza sulla ottimizzazione dell'attuale capacità di funzionare; piuttosto assume che il sistema cognitivo sia sopraffatto dall'incertezza ambientale e si ritrovi perciò con il problema di costituire le condizioni del proprio esercizio, ossia di ripristinare/sviluppare il valore delle risorse semiotiche a fondamento del proprio fondamento.

La distinzione tra problem solving e problem setting può aiutare ad evidenziare la differenza tra le due ipotesi. Nel caso del problem solving, il soggetto deve interrompere la procedura consolidata, l'insistere con la quale produrrebbe fissità funzionale, e mappare lo spazio del problema al fine d'identificare una nuova organizzazione degli elementi che risulti operare da soluzione appropriata. Per fare questo, il soggetto deve affidarsi a regole di second'ordine (euristiche) che aumentano la funzionalità dei processi cognitivi, per mezzo del potenziamento del controllo metacognitivo. Il processo è lo stesso nel caso del problem setting, tuttavia ad un livello logico più alto - infatti, in questo caso, differentemente da quanto accade nel problem solving, il soggetto non può fare affidamento su una rappresentazione del problema come già disponibile, dunque operante come base per la costruzione metacognitiva dello spazio del compito; piuttosto, è la rappresentazione stessa del problema che va costituita - i.e. configurata come risultato di una interpretazione innovativa dello stato delle cose.

Si potrebbero fornire svariati convincenti esempi di come l'incapacità di distinguere tra domanda di ottimizzazione funzionale (i.e. la domanda implicita nella visione delle competenze come modo di adattarsi a contesti dinamici) e 
domanda di ripristino di senso (i.e. la domanda implicita nella visione delle competenze come modo di adattarsi a contesti generativi di perdita di senso) sia alla radice del fallimento di molte politiche in ambito educativo (e.g. Matsupolous et al., 2019) così come in altri ambiti (Andriola et al., 2019).

Un'ultima osservazione. L'ipotesi della perdita di senso non è alternativa all'ipotesi dell'aumentata dinamicità; la riduzione di incertezza implicata nella prima è la pre-condizione per l'ottimizzazione metacognitiva prevista dalla seconda. D'altra parte, le due ipotesi sono associate con setting diversi - infatti, nella terminologia adottata nel presente lavoro, laddove l'aumentata dinamicità richiede un setting secondario (i.e. un setting che vincola il sistema cognitivo ad un criterio di appropriatezza alla contingenza ambientale), l'ipotesi della perdita di senso suggerisce il bisogno di un setting terziario, al fine di abilitare gli studenti alla elaborazione dell'innovazione semiotica richiesta per far fronte alla incertezza radicale.

\section{Esempi di setting educativo terziario}

In questo paragrafo conclusivo delineiamo due dispositivi che esemplificano l'idea di istruzione universitaria come setting terziario.

Laboratorio riflessivo. In ogni contesto accademico gli studenti sono coinvolti in attività laboratoriali. Solitamente, queste attività sono mirate a sviluppare capacità pratiche e/o a fornire uno spazio per esplorare la relazione tra apprendimenti e problemi/azioni. Il laboratorio riflessivo è progettato con un diverso proposito - fornire un setting di piccolo gruppo di sospensione dell'attività formativa finalizzata, che mette i partecipanti nella condizione di comunicare la propria esperienza del ruolo di studente (e.g. le teorie e aspettative suo ruolo, le fantasie, le credenze e le proiezioni sul futuro). Ciò non è fatto né come un fine in sé (come sarebbe se fosse inteso in termini di setting primario), né per scambiare informazioni e ricercare soluzioni funzionali alle criticità riportate (come sarebbe se fosse inteso in termini di setting secondario); piuttosto, le produzioni narrative sono analizzate al fine di comprendere i presupposti di base su cui si fondano (i.e. la cultura organizzativa che esprimono) e di usare tale comprensione per aiutare gli studenti a potenziare la propria capacità di dare senso all'esperienza (per una presentazione di questo approccio, vedi Caviglia \& Salvatore, 1992).

L'esplorazione del servizio. Il modello "service learning" ha guadagnato credito nel contesto dell'istruzione universitaria. Tale modello combina la visione dell'apprendere tramite il fare (learning by doing) con il principio pedagogico della responsabilità verso la comunità. Con "esplorazione del servizio" 
intendiamo un setting di insegnamento-apprendimento che assume il format del service leraning, tuttavia in funzione di una cornice metodologica parzialmente differente. Più specificamente, il concetto di "esplorazione del servizio" è basato sulla teoria manageriale del servizio. Nel contesto di quella letteratura (e.g. Norman, 1981), il concetto di servizio denota un peculiare modello di relazione produttore-cliente basata su tre caratteristiche principali: a) Prosumership: produttore e consumatore cooperano nella costruzione del valore, perciò il produttore non ha il controllo totale sul processo di produzione, dipendendo quest'ultimo anche dal cliente (come succede nell'insegnamento-apprendimento, ad esempio); b) Appropriatezza: il valore del prodotto non risiede nel risultato in se stesso, ma nella capacità di tale risultato di risultate utile al cliente, ossia nella sua capacità di potenziare l'abilità del cliente di perseguire gli obiettivi motivanti la richiesta del servizio; c) Dinamicità: la relazione produttore-cliente evolve nel tempo, attraverso e nei termini di cambiamenti ricorsivi che ambedue attivano nell'altro.

Queste tre caratteristiche implicano una peculiare struttura cognitiva nel produttore, al quale è richiesto di de-assolutizzare il proprio punto di vista e di mappare il punto vista del cliente, al fine di ottimizzare le condizioni della cooperazione con quest'ultimo, così come d'identificare le forme di utilità (e.g. utilità di tempo, spazio, costi, simbolica) che possono operare da vettore del valore del servizio (Salvatore, Gennaro et al., 2019). In ragione di questa visione, l'esplorazione del servizio è un dispositivo mirato a mettere gli studenti nella condizione di entrare in rapporto con i propri apprendimenti, nel contesto della capacità di tali apprendimenti di regolare una relazione di servizio. In altre parole, per mezzo di questo setting lo studente può esplorare le implicazioni della costruzione di uno scambio cooperativo (i.e. lo scambio richiesto per portare a termine il servizio) con l'estraneo (il cliente). Queste implicazioni concernono la necessità di relativizzare i propri assunti (e.g. assunti riguardanti l'identità professionale) così come il formato e la modalità di utilizzo della conoscenza al fine di renderli appropriati alla ricerca di utilità del cliente. In breve, il servizio sfida l'autoreferenzialità dell'apprendimento, stimolando gli studenti ad introdurre la mappatura del punto di vista del cliente come un fondamentale regolatore dei loro processi cognitivi.

È bene osservare che nessuno dei due dispositivi proposti sopra sono in se stessi originali; è il modo di utilizzarli, la cornice metodologica e concettuale fondante la loro finalizzazione, che li rende specifici. In questa prospettiva, $\mathrm{i}$ dispositivi proposti condividono la qualità fondamentale di essere pratiche riflessive. Come qui intesa, la valenza di riflessività di tali pratiche risiede in questo: i dispositivi sopra proposti sono attività che, come ogni altra attività del contesto formativo, istanzia il sistema di assunti (i.e. credenze generalizzate, schemi interpretativi, universi simbolici) fondanti e regolanti la partecipazione 
degli studenti al contesto dell'insegnamento-apprendimento. Allo stesso tempo tuttavia, il sistema di assunti non è attuato come framework normativo dato, regolativo dell'attività formativa; differentemente dalle altre attività formative volte a promuovere competenze funzionali (i.e. pensiero critico, auto-regolazione, capacità di apprendere), la pratica riflessiva opera con l'obiettivo di sospendere-mettere tra parentesi, per usare la terminologia della fenomenologia - la funzione normativa del sistema di significati incorporato in essa, al fine di renderlo oggetto di analisi. I principali risultati dell'apprendimento che tale procedura riflessiva si propone di produrre sono: a) riconoscere il proprio sistema di assunti e il loro ruolo nel vincolare e canalizzare la partecipazione al contesto formativo; b) rivedere/sviluppare il sistema di assunti nei suoi aspetti critici per il processo formativo; c) apprendere a de-assolutizzare i propri assunti come metodo per generare innovazione.

In ultima analisi, i dispositivi delineati sopra non producono nessuna competenza funzionale; piuttosto, seguono la regola paradossale di - se così si può dire - svuotare il processo formativo del suo proposito produttivo ed usare tale proposito come pretesto attraverso cui mettere in primo piano il sistema latente di assunti da sottoporre ad analisi. In breve, questi dispositivi sono pratiche ricorsive di terzietà applicate ai propri sistemi di assunti, mirate a promuovere la posizione di estraneità dello studente rispetto a se stesso, dunque la possibilità creativa di innovazione che tale posizione è in grado di elicitare.

In un mondo dove l'altro è sempre più spesso sinonimo di nemico, la competenza a relazionarsi con la propria estraneità si propone come risorsa semiotica preziosa, che l'istruzione universitaria può contribuire a promuovere e disseminare.

\section{Conclusioni}

In questo articolo abbiamo proposto un'analisi dell'impatto che le profonde turbolenze socio-istituzionali che attraversano le società occidentali contemporanee esercitano sulla capacità delle persone di dare senso all'esperienza. Abbiamo successivamente evidenziato le implicazioni di tale analisi, relativamente alla funzione che il sistema dell'istruzione, in particolare l'istruzione universitaria, può assolvere in tale scenario.

I punti principali di tale discussione possono essere sintetizzati come segue.

a) La diffusione dell'affettivizzazione nelle società occidentali è il modo - il più diretto e potente - con cui le persone fronteggiano la condizione di incertezza radicale cui sono esposte, come risultato dell'aumento esponenziale di dinamicità che caratterizza le società contemporanee. 
b) Più specificamente, la dinamicità implica l'indebolimento dei confini che articolano il campo sociale; a sua volta, tale processo di indebolimento determina il decadimento di ciò che abbiamo proposto di definire il valore semiotico dei segni, vale a dire la loro capacità di alimentare l'elaborazione del senso dell'esperienza da parte del soggetto;

c) L'affettivizzazione è un modo efficace di contrastare il decadimento del valore semiotico in quanto gli affetti sono segni con una struttura semiotica bipolare e generalizzata; grazie a tale struttura, sono comunque in grado di fornire un'interpretazione "embodied" del campo dell'esperienza; in altri termini, mantengono valore indipendentemente dalle contingenze ambientali.

d) Riconosciuta tale funzione di sopravvivenza dell'affettivizzazione, va allo stesso modo riconosciuto che la sua adozione come modo fondamentale di relazione con la sfera pubblica genera effetti critici, in quanto si traduce in interpretazioni semplificate della realtà che non sono in grado di fondare ed alimentare progresso civile e sociale.

e) È stato suggerito che la diffusione dell'affettivizzazione della sfera pubblica può essere contrastata non solo riducendo le fonti d'incertezza che la alimentano, ma anche attraverso una strategia mirante a potenziare la capacità cognitiva dei soggetti di fronteggiare l'incertezza data.

f) Questa strategia consiste nel promuovere risorse semiotiche innovative (nuovi significati, codici, istituzioni) che siano in grado di difendere l'attività interpretativa dal decadimento del suo valore, attraverso la costituzione di relazioni più complesse e astratte tra gli elementi dell'esperienza, piuttosto che attraverso la semplificazione affettiva - vale a dire attraverso la costituzione di relazioni omogeneizzate basate su significati corporei.

g) Mentre il modo affettivo di affrontare l'incertezza è una modalità pronta all'uso cui gli individui sono soliti ricorre dal primo momento in cui vengono alla vita, l'innovazione semiotica è una strategia cognitiva che richiede specifiche condizioni di contesto. In particolare, essa funziona nella misura in cui è inscritta in una pratica sociale, ossia nella misura in cui una determinata interazione sociale adotta l'innovazione semiotica come propria forma immanente di regolazione.

h) Abbiamo definito come setting terziario questo tipo di pratiche sociali. I setting terziari sono caratterizzati dal fatto che il loro funzionamento è subordinato ad un ambiente dinamico. Di conseguenza, il setting terziario è una condizione continua di decadimento locale del valore del sistema di significato che lo fonda; ciò significa che il setting terziario è impegnato in una operazione ricorsiva di aggiornamento/sviluppo del sistema di significato volta a "rincorrere" le dinamiche ambientali. 
i) L'istruzione, e più in particolare l'istruzione universitaria, può - dovrebbe funzionare come un setting terziario, incubatore di innovazione semiotica. Tale prospettiva ha implicazioni rilevanti per il modo di concepire e implementare le prassi di insegnamento-apprendimento, così come per il modo di configurare gli ambienti didattici.

\section{Bibliografia}

Andriola V., Been W., Cremaschi M., Fini V., Matsopoulos A., Willet J., Salvatore S. (2019). Policies and Sensemaking. In: Salvatore S., Fini V., Mannarini T., Valsiner J., Veltri G. A. (Eds.). Symbolic Universes in Time of (Post)Crisis. Crisis. The Future of European Societies (pp. 271-291). Cham (Switzerland): Springer

Barrett Feldman. L. (2006). Solving the emotion paradox: Categorization and the experience of emotion. Personality and Social Psychology Review, 10: 20-46.

Barsalou L. W. (1999). Perceptual symbol systems. Behavioural and Brain Science, 22, 577-609.

Barsalou L. W. (2011). Integrating Bayesian analysis and mechanistic theories in grounded cognition. Behavioural and Brain Science, 34: 191-192.

Barsalou L. W. (2016). Situated conceptualization: Theory and applications. In: Coello Y., Fischer M. H. (Ed.). Foundations of embodied cognition: Perceptual and emotional embodiment (pp. 11-37). New York: Routledge/Taylor \& Francis.

Battaglia R. (1953). Storia della resistenza italiana. Torino: Einaudi.

Bauman Z., \& Bordoni C. (2014). State of crisis. Cambridge: Polity Press.

Berger P. L., \& Luckmann T. (1966). The social construction of reality. London: Penguin Books.

Bertini M. (2012). Psicologia della salute. Milano: Raffaello Cortina Editore.

Borghi A. M., Binkofski F., Castelfranchi C., Cimatti F., Scorolli C. Tummolini L. (2017). The Challenge of Abstract Concepts. Psychological Bulletin, 143(3): 263292.

Bruner J. (1990). Acts of Meaning. Cambridge Mass.: Harvard University Press.

Carli R., \& Paniccia R. M. (1999). Psicologia della formazione [Psychology of the Training]. Bologna: il Mulino.

Carli R., \& Salvatore S. (2001), L'immagine dello psicologo [The image of the psychologist]. Roma: Kappa Editore.

Cavalli A., \& de Lillo A. (a cura di) (1993). Giovani anni '90. Terzo rapporto Iard sulla condizione giovanile in Italia. Bologna: il Mulino.

Caviglia G., \& Salvatore S. (1992). Riflessioni sulla formazione in psicologia: le Esperienze Pratiche Guidate. Lo Psicologo, 2(1): 51-70.

Codagnone C., Bogliacino F., \& Veltri G. A. (2018). Scienza in vendita: Incertezza, interessi e valori nelle politiche pubbliche. Milano: Egea.

Cole M. (1996). Cultural Psychology. A once and future discipline. Cambridge Mass.: Harvard University Press. 
Council of the Eurpoean Union (2018). Recommendation of the Council of the European Union, 22 May 2018 on key competences for lifelong learning. https://eurlex.europa.eu/legal-content/EN/TXT/?uri=uriserv\%3AOJ.C_.2018.189.01.0001.01.ENG\&toc=OJ\%3AC\%3A2018\%3A189\%3A TOC.

Cuccio V., Gallese V. (2018). A Peircean account of concepts: grounding abstraction in phylogeny through a comparative neuroscientific perspective. Philosophical Transaction of the Royal Society. Biological Sciences, 373.

Durkheim E. (1893/1984). The Division of Labour in Society. New York: Free Press. (original work in French, 1893).

Engel A. K., Friston K. J., Kragic D. (2015). Introduction: Where's the Action? In Engel A. K., Friston K. J., Kragic D. (Ed.). The Pragmatic Turn. Toward ActionOriented Views in Cognitive Science (pp. 1-15). Cambridge (MASS): MIT.

Gallagher S. (2005). How the Body shapes the mind. Oxford: Oxford University Press.

Gibson (1979). The Ecological Approach to Visual Perception. Boston: Houghton Mifflin.

Gonçalves M. M., Ribeiro A. P., Stiles W. B., Conde T., Matos M., Martins C., \& Santos A. (2011). The role of mutual in-feeding in maintaining problematic selfnarratives: Exploring one path to therapeutic failure. Psychotherapy Research, 21(1): 27-40. doi: 10.1080/10503307.2010.507789.

Greenberg J., \& Arndt J. (2012). Terror management theory. In: Van Lange P. A. M. K. Higgins, E. T. (Ed.), Handbook of theories of social psychology (pp. 398-415). Thousand Oaks (CA) US: Sage Publications Ltd.

Husserl E. (1991). On the Phenomenology of the Consciousness of Internal Time (18931917). Collected Works (J. B. Brough, Trans. Vol. 4). Dordrecht: Kluwer Academic.

Kay A. C., Whitson J. A., Gaucher D., Galinsky A. D. (2009). Compensatory Control. Achieving Order Through the Mind, Our Institutions, and the Heavens. Current Direction of Psychological Science, 18(5), 264-268.

Klein M. (1967). Contribution to Psychoanalysis, 1921-1945. New York: Mac GrawHill.

Lindblom J. (2015). Embodied Social Cognition. Heidelberg: Springher

Major B., Kaiser C. R., O’Brien L. T., McCoy S. K. (2007). Perceived Discrimination as Worldview Threat or Worldview Confirmation: Implications for Self-Esteem. Journal of Personality and Social Psychology, 92(6): 1068-1086.

Mannarini T., Salvatore S. (2019). Making sense of ourselves and others: A contribution to the community-diversity debate. Community Psychology in Global Perspective. 5(1): 26-37

Marigold D. C., McGregor I., Zanna M P. (2010). Defensive Conviction as Emotion Regulation: Goal Mechanisms and Interpersonal Implications. In Arkin R. M., Oleson K. C., Carroll P. J. (Ed.). Handbook of the Uncertain Self (pp. 232-248). Hove, UK: Psychology Press.

Matsopoulos A., Griva A.-M., Psinas P., Monasterioti I. (2019). Teachers' Perceptions of Teacher Evaluation, Professional Identities and Educational Institutions: An Analysis Based on the Symbolic Universes Approach. In: Salvatore S., Fini V., 
Mannarini T., Valsiner J., Veltri G. A. (Eds.). Symbolic Universes in Time of (Post)Crisis. Crisis. The Future of European Societies (pp. 191-214). Cham (Switzerland): Springer.

Maturana M. R., \& Varela J. F. (1980). Autopoiesis and Cognition. The Realization of the Living. Dordrecht, NL: Reidel Publishing Co.

Mazzoni G. (2015). I destini generali. Bari: Laterza Editore.

Montesarchio G. Crotti M. T. (1993). Dal riconoscimento al disconoscimento. Rivista di Psicologia Clinica, 7(1-2): 169-174.

Mudde C. (2004). The Populist Zeitgeist. Government and Opposition, 39(4): 542-563.

Norman R. (1986). Service management: Strategy and leadership in service businesses. New York: Wiley \& Sons

Osgood C. E., May W. H., Miron M. S. . (1975). Cross-cultural Universals of Affective Meaning. Chicago: Illinois University Press.

Osgood C. E., Suci G. J., \& Tannenbaum P. H. (1957). The Measurement of Meaning. Chicago, ILL: University of Illinois Press

Paniccia R. M. (2003). The school client as an unknown friend: a stranger. European Journal of School Psychology, 1(2): 247-285.

Peirce C. S. (1932). On Sign. In C. Hartshorne, \& P. Weiss (Eds.), Collected Papers of Charles Sanders Peirce (Volume II). Cambridge, MA: Harvard University Press [Original version: 1897].

Piketty T. (2014). Capital in the twenty-first century. Cambridge, MA: Harward University Press (original work: Editions du Seuil, Paris, 2013).

Proulx T., \& Inzlicht M. (2012). The Five “A”s of Meaning Maintenance: Finding Meaning in the Theories of Sense-Making. Psychological Inquiry, 23(4): 317-335. doi: 10.1080/1047840X.2012.702372.

Rumiati R. I., Ciolfi A. Di Benedetto A., Sabella M., Infurna M. R. Ancaiani A., Checchi D. (2018). Key-competences in higher education as a tool for democracy. Form@are.Open Journal per la formazione in rete, 1-13.

Salvatore S. (2012). Social Life of the Sign: Sensemaking in Society. In: Valsiner J. (Eds). The Oxford Handbook of Culture and Psychology (pp. 241-254). Oxford: Oxford University Press.

Salvatore S. (2016). Psychology in black and white. The project of a theory-driven science. Charlotte, NC: InfoAge Publishing.

Salvatore S. (2018). Cultural Psychology as the Science of Sensemaking: A Semioticcultural Framework for Psychology. In: Rosa A. \& Valsiner J. (Eds). The Cambridge Handbook of Sociocultural Psychology, 2nd Edition (pp 35-48). Cambridge: Cambridge University Press.

Salvatore S. (2019, on line first). Beyond the Meaning Given. The Meaning as Explanandum. Integrative Psychological and Behavioural Science.

Salvatore S., Fini V., Mannarini T., Valsiner J. Veltri G. A. (Eds, 2019). Symbolic Universes in Time of (Post)Crisis. The Future of European Societies. Cham (Switzerland): Springer.

Salvatore S., Fini V., Mannarini T., Veltri G. A., Avdi E., Battaglia F., Castro-Tejerina J., Ciavolino E., Cremaschi M., Kadianaki I., Kharlamov A. N., Krasteva A., Kullasepp K., Matsopoulos A., Meschiari C., Mossi P., Psinas P., Redd R., Rochira 
A., Santarpia A., Sammut G., Valsiner J., \& Valmorbida A. (2018) Symbolic universes between present and future of Europe. First results of the map of European societies' cultural milieu. PLoS ONE, 13(1): e0189885.

Salvatore S., \& Freda M. F. (2011). Affect Unconscious and Sensemaking. A Psychodynamic Semiotic and Dialogic Model. New Ideas in Psychology, 29: 119-135.

Salvatore S., Gennaro A., Manfreda A., Calogiuri S. (2019). Models of value construction. For a semio-dialectical approach to organization and social action. In: Bendassoli P. F. (Ed.). Culture, work and psychology: Invitations to dialogue. Charlotte (NC): Information Age Publishing

Salvatore S., Mannarini T., Avdi E., Battaglia F., Cremaschi M., Forges Davanzati G., Fini V., Kadianaki I., Krasteva A., Matsopoulos A., Mølholm M., Redd R., Rochira A., Russo F., Santarpia A., Sammut G., Valmorbida A., Veltri G. A. 2018, on line first). Globalization, demand of sense and enemization of the other. A psycho-cultural analysis of European societies' socio-political crisis. Culture \& Psychology.

Salvatore S., Marsico P., Andrisano-Ruggieri (2015). Psychology of Reflexivity and reflexivity for psychology. In: Marsico P., Salvatore S., Andrisano-Ruggieri R. (2015). Reflexivity and Psychology. Yearbook of Idiographic Science Series Vol. 6 (pp. vii-xxi). Charlotte NC: InfoAge Publishing

Salvatore S., \& Scotto di Carlo M. (2002). Domanda sociale e missione educativa. Psicologia Scolastica, 1(1): 101-131

Salvatore S., \& Venuleo C. (2017). Liminality in semiotic key. The mutual in-feeding recursion of absence and presence. Theory \& Psychology, 13: 1-16

Salvatore S., \& Zittoun T. (Eds.) (2011a). Outlines of a psychoanalytically informed cultural psychology. In: Salvatore S., \& Zittoun T. (Eds). Cultural Psychology and Psychoanalysis in Dialogue. Issues for Constructive Theoretical and Methodological Synergies (pp. 3-46). Charlotte, NC: Information.

Salvatore S., Valsiner J. Veltri G. A. (2019). The Theoretical and Methodological Framework. Semiotic Cultural Psychology, Symbolic Universes and Lines of Semiotic Forces. In: Salvatore S., Fini V., Mannarini T., Valsiner J., Veltri G. A. (Eds). Symbolic Universes in Time of (Post)Crisis. The Future of European Societies (pp. 25-49). Cham (Switzerland): Springer.

Sedikides C., De Cremer D., Hart C. M., Brebels L. (2010). Procedural Fairness Responses in the Context of Self-Uncertainty. In: Arkin R. M., Oleson K. C., Carroll P. J. (Ed.). Handbook of the Uncertain Self (pp. 142-160). Hove, UK: Psychology Press.

Stenner P. \& Moreno-Gabriel E. (2013). Liminality and affectivity: The case of deceased organ donation, Subjectivity, 6(3): 229-253. doi:10.1057/sub.2013.9.

Stern D. N. (2004). The present moment in psychotherapy and everyday life. New York: W. W. Norton \& Co.

Tonti M., Salvatore S. (2015). The Homogenization of Classification Functions Measurement (HOCFUN): A method for measuring the salience of emotional arousal in thinking. American Journal of Psychology, 128(4): 469-483.

United Nations Development Programme (2016). 2016 Human Development Report. Human Development for Everyone. New York.

Valsiner J. (2014). An invitation to cultural psychology. London: Sage Publications. 
van Den Bos K., Lind A. (2010). The Social Psychology of Fairness and the Regulation of Personal Uncertainty. In: Arkin R. M., Oleson K. C., Carroll P. J. (Ed.). Handbook of the Uncertain Self (pp. 122-141). Hove, UK: Psychology Press.

Varela J. F., \& Depraz N. (2000). At the source of time: Valence and the constitutional dynamics of affect. Arobase, 4(1-2): 143-66.

Veltri G. A., Redd R., Mannarini T., \& Salvatore S. (2019). The identity of Brexit: A cultural psychology analysis. Journal of Community and Applied Social Psychology, 29(1): 18-31.

Verheggen T., \& Baerveldt C. B. (2007). “We Don't Share!” Exploring the theoretical ground for social and cultural psychology: The social representation approach versus an enactivism framework. Culture \& Psychology, 13(1): 5-27.

Vygotsky L. S. (1978). Mind in society. Cambridge Mass.: Harvard University Press.

Weary G., Tobin S. J., Edwards J. A. (2010). The Causal Uncertainty Model Revisited. In: Arkin R. M., Oleson K. C., Carroll P. J. (Ed.). Handbook of the Uncertain Self (pp. 78-100). Hove, UK: Psychology Press.

Winnicott D. W. (1952). Psychoses and child care. In: Winnicott D. W. (1975), Through pediatrics to psychoanalysis: Collected papers (pp. 219-228). New York: Basic Books.

Wittgenstein L. (1958). Philosophical Investigations. Oxford: Basil Blackwell (Original work published in 1953).

World Bank (2015). World development report 2015: Mind, society, and behavior. Washington, DC: World Bank.

Zinola A. (2016, 4 gennaio). Quanti sono (davvero) gli stranieri in Italia. La Citta Nuova, Corriere della Sera, http://lacittanuova.milano.corriere.it/2016/01/04/quanti-sono-gli-stranieri-in-italia-dati-veri-e-visione-distorta/?refresh ce-cp.

Znaniecki F., \& Thomas W. I. (1918-1920). The Polish Peasant in Europe and America. Chicago: Chicago University Press. 